\title{
Valve-sparing root replacement and composite valve graft replacement in patients with aortic regurgitation: From the Japan Cardiovascular Surgery Database
}

\author{
Takashi Kunihara, MD, PhD, ${ }^{\mathrm{a}}$ Nao Ichihara, MD, PhD, MPH, ${ }^{\mathrm{b}}$ Hiroaki Miyata, $\mathrm{PhD},{ }^{\mathrm{b}}$ \\ Noboru Motomura, MD, PhD,${ }^{\mathrm{c}}$ Kenichi Sasaki, MD, ${ }^{\mathrm{d}}$ Minoru Matsuhama, MD, ${ }^{\mathrm{e}}$ and \\ Shinichi Takamoto, MD, $\mathrm{PhD},{ }^{\mathrm{c}}$ for the Japan Cardiovascular Surgery Database
}

\section{ABSTRACT}

Objectives: The advantage of valve-sparing root replacement (VSRR) over aortic root replacement with a composite valve graft $(\mathrm{CVG})$ remains unclear. We compared these 2 procedures with regard to early outcomes with propensity score matching using the Japan Cardiovascular Surgery Database.

Methods: Of 5303 patients from the Japan Cardiovascular Surgery Database who had undergone aortic root replacement in 2008 to 2017, emergent/urgent or redo cases and those with infective endocarditis or aortic stenosis were excluded (included $\mathrm{n}=3841$ ). Two propensity score-matched groups treated with VSRR or CVG replacement $(\mathrm{n}=1164 \mathrm{each})$ were established.

Results: Overall, VSRR was more frequently performed for younger patients with Marfan syndrome with lower operative risk and aortic regurgitation grade compared with CVG replacement. After matching, a weaker but similar trend still existed in baseline characteristics. Although more concomitant procedures were performed in the CVG group, myocardial ischemia and cardiopulmonary bypass time was significantly longer in the VSRR group (median, 193 and 245 minutes) than the CVG group (172 and 223 minutes, both $P<.01$ ). The CVG group was associated with a significantly greater incidence of postoperative stroke $(2.5 \%$ vs $1.1 \%, P=.01)$ and prolonged ventilation $>72$ hours $(7.0 \%$ vs $4.6 \%$, $P=.02)$. In-hospital mortality rates were significantly greater in the $\mathrm{CVG}$ group $(1.8 \%)$ than the VSRR group $(0.8 \%, P=.02)$.

Conclusions: In overall Japanese institutions, VSRR was more frequently performed for patients at low risk and was associated with better morbidity and mortality rates than CVG replacement. After matching, VSRR was also associated with better morbidity and mortality rates despite longer procedure time. (J Thorac Cardiovasc Surg 2019;158:1501-11)

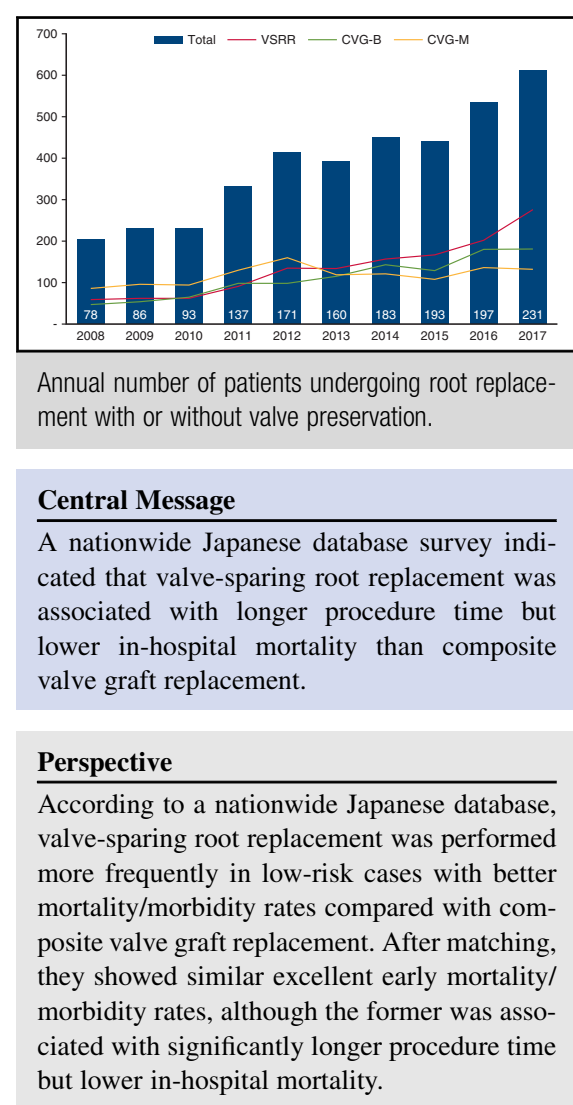

See Commentaries on pages 1512 and 1514.

\footnotetext{
From the a Department of Cardiac Surgery, The Jikei University School of Medicine, Tokyo, Japan; ${ }^{\mathrm{b}}$ Department of Healthcare Quality Assessment, University of Tokyo Graduate School of Medicine, Tokyo, Japan; 'Japan Cardiovascular Surgery Database, Tokyo, Japan; ${ }^{\mathrm{d}}$ Department of Cardiovascular Surgery, Saitama Sekishinkai Hospital, Sayama, Japan; and ${ }^{\mathrm{e}}$ Department of Cardiovascular Surgery, the Cardiovascular Institute, Tokyo, Japan.

Received for publication May 26, 2018; revisions received Jan 5, 2019; accepted for publication Jan 31, 2019; available ahead of print April 2, 2019.

Address for reprints: Takashi Kunihara, MD, PhD, Department of Cardiac Surgery,

The Jikei University School of Medicine, 3-25-8 Nishishinbashi, Minato-ku, Tokyo, 105-8461, Japan (E-mail: kunihara@jikei.ac.jp).

$0022-5223 / \$ 36.00$

Copyright (c) 2019 by The American Association for Thoracic Surgery

https://doi.org/10.1016/j.jtcvs.2019.01.122
}

Surgical intervention for aortic root aneurysm with or without aortic valve regurgitation (AR) was first reported by Wheat and colleagues in $1964 .{ }^{1}$ However, future potential expansion of the remnant aortic wall was a matter of great concern in their technique. Since Bentall and de Bono

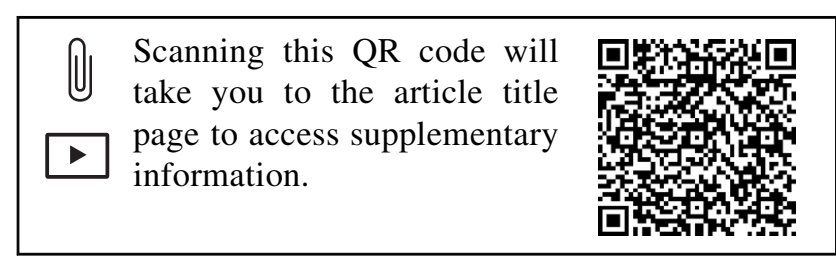




\section{Abbreviations and Acronyms \\ $\mathrm{AR}=$ aortic valve regurgitation \\ $\mathrm{CABG}=$ coronary artery bypass grafting \\ $\mathrm{CVG}=$ composite valve graft \\ CVG-B $=$ biological composite valve graft \\ CVG-M = mechanical composite valve graft \\ JCVSD = Japan Cardiovascular Surgery Database \\ MFS = Marfan syndrome \\ SMD $=$ standardized mean difference \\ VSRR = valve-sparing root replacement}

advocated total replacement of the aortic root with a composite valve graft (CVG) in $1968,{ }^{2}$ this has become the gold standard treatment method, referred to as the Bentall procedure. However, the majority of patients are relatively young, so the requirement for lifelong anticoagulation has emerged as another concern. The Ross procedure using an autologous pulmonary valve can eliminate the need for anticoagulation and provides durable long-term outcome; however, the high degree of operative invasiveness and future potential risk of reoperation associated with the use of a valved conduit in the pulmonary position remain inherent disadvantages of this method. Stentless bioprosthesis or biological composite valve graft (CVG-B) can also eliminate the need for anticoagulation but are not recommended for young patients because of their short durability.

The majority of cases with root dilatation have aortic cusps, which may be stretched but not degenerated, and can theoretically be preserved when aortic root replacement is indicated. Technical developments over the past 25 years have resolved this dilemma with 2 types of valve-sparing root replacement (VSRR): reimplantation of the aortic valve (hereinafter referred to as reimplantation) proposed by $\mathrm{Da}-$ vid and Feindel in $1992^{3}$ and remodeling of the aortic root (hereinafter referred to as remodeling) advocated by Sarsam and Yacoub in 1993. ${ }^{4}$ Although they both have a number of advantages and disadvantages, these procedures can eliminate the need for anticoagulation and provide physiological hemodynamics through technical refinements and therefore are associated with good long-term outcome. ${ }^{5,6}$

The numbers of VSRR and Bentall procedures using CVG-B have increased, whereas those of Bentall procedures using mechanical composite valve graft (CVG-M) have decreased in the United States. ${ }^{7,8}$ Each procedure has been shown to provide acceptable early outcomes. However, details regarding the advantages or disadvantages of one procedure over the others have not exactly been reported. With the exception of only one single-institution study comparing small numbers of cases using propensity score matching, the majority of reports compared inhomogeneous cohorts. ${ }^{8}$ The present study was performed to compare clinical outcomes associated with VSRR and the Bentall procedure using propensity score-adjusted analysis based on the nationwide Japan Cardiovascular Surgery Database (JCVSD).

\section{PATIENTS AND METHODS}

The JCVSD was established in 2000 to evaluate outcomes of cardiovascular surgery in Japan by prospectively collecting more than 300 variables from 581 institutions (as of October 2017). The number of participating institutions registering at least $1 \mathrm{VSRR}$ or CVG replacement case per year over time is indicated in Figure 1. The institutional review board at each institution has approved the data collection for the JCVSD, and the Data Utilization Committee of the JCVSD approved the use of data for the current study (\#A0063). Patients have been given opt-out participant information instead of giving written informed consent based on the ethical policy of National Clinical Database (http://www.ncd.or.jp/about/ethical_considerations. html). The further detailed information about the JCVSD has previously been described ${ }^{\mathrm{E} 1, \mathrm{E} 2}$ and available online at http://www.jacvsd.umin.jp.

We first accessed the JCVSD records and identified 5303 surgical cases of elective aortic root replacement using either a valve-sparing method $(\mathrm{n}=1518)$ or a composite valve graft $(\mathrm{n}=3785)$ between 2008 and 2017. Cases of redo surgery $(n=1089)$ and those of patients with infective endocarditis $(n=186)$ or aortic stenosis $(n=403)$ were excluded. There were still many inhomogeneities between patients undergoing VSRR $(\mathrm{n}=1345)$ and CVG replacement $(\mathrm{n}=2496)$ (Table 1$)$. Therefore, we established a propensity score-matched sample $(\mathrm{n}=1155$ each) in which to compare the outcomes of VSRR and CVG replacement (Figure 2 and Table 1). The distributions of the year of the operations and operative procedures are shown in Tables 1 and 2. The number of operations increased each year with the increased number of participating institutions over time (Figure 1). Since 2014, the number of CVG-B procedures has exceeded that of CVG$\mathrm{M}$. Among VSRR cases, remodeling and reimplantation have been distinguished in the database only since 2017. In 2017, remodeling and reimplantation were performed in 109 of $276(39.5 \%)$ and 167 of 276 cases $(60.5 \%)$, respectively. The geometric size of the proximal aorta has been recorded only since 2013 and morphology of the aortic valve (eg, bicuspid aortic valve) only since 2016. Since 2013, maximum diameter in the short axis of the proximal aorta was less than $40 \mathrm{~mm}$ in $38(1.9 \%), 40$ to $49 \mathrm{~mm}$ in $509(24.8 \%), 50$ to $59 \mathrm{~mm}$ in $973(47.4 \%)$, and $60 \mathrm{~mm}$ or more in 534 cases $(26.0 \%)$ among 2054 cases with valid record of the diameter. Since 2016, 17 unicuspid (1.5\%), 126 bicuspid (11\%), 993 tricuspid (87\%), and 2 quadricuspid $(0.2 \%)$ aortic valves were registered (unknown: $9,0.8 \%$ ) among 1147 cases. These partially available variables were not included in the propensity score matching.

\section{Propensity Score Matching}

We used propensity score matching to account for covariates that may have influenced whether VSRR or CVG replacement was selected for treatment. Each patient's estimated propensity score, which is the likelihood that the patient would be treated by VSRR, was calculated by a multivariable logistic model using preselected 29 preoperative variables as independent variables, as described in Appendix E1. Interaction terms was used only between Marfan syndrome (MFS) and age. We then matched patients who underwent VSRR with those who underwent CVG replacement in a 1:1 ratio using greedy (nearest neighbor) matching with a caliper width equal to 0.25 standard deviation of the propensity score (0.050688) (Cohort A). Mirror histograms before and after matching of the propensity scores in each group are shown in Figure E1.

\section{Statistical Analysis}

In most variables, the proportion of missing values was less than $0.1 \%$, although some variables were recorded only for cases in the later part of the 


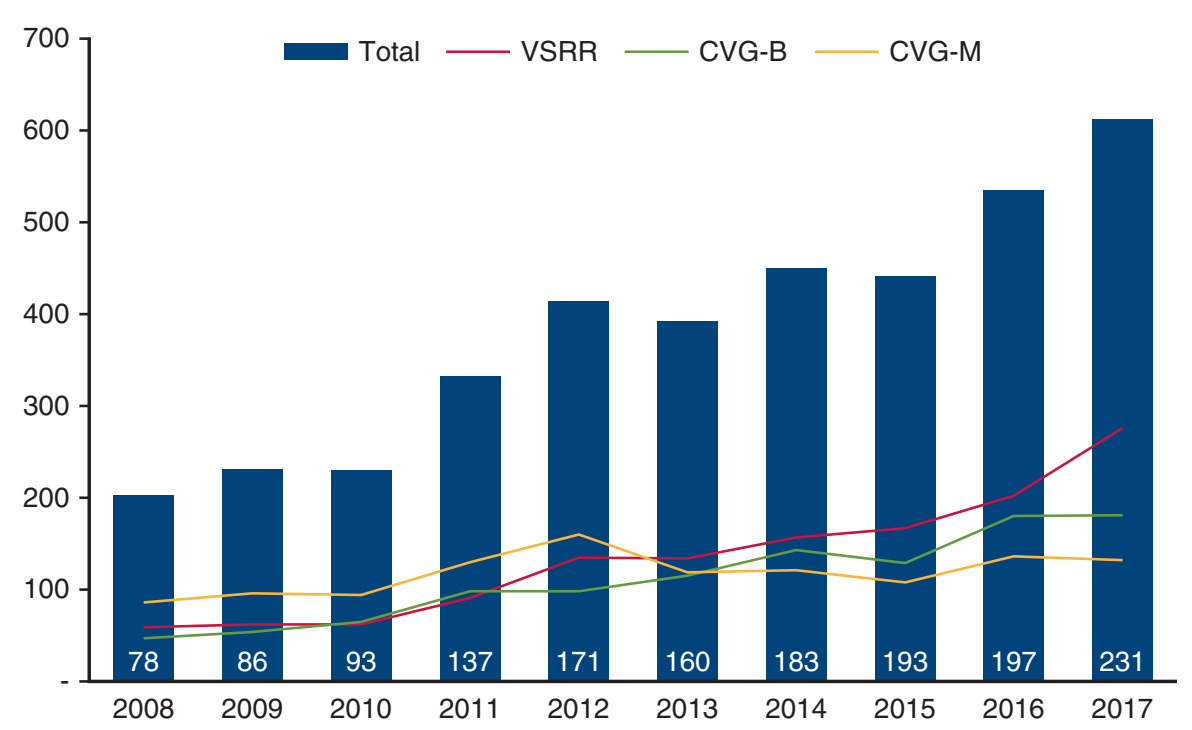

FIGURE 1. Annual distribution of the cases undergoing aortic root replacement according to the operative procedures and the number of participating institutions registering at least 1 aortic root replacement case per year (white numbers). VSRR, Valve-sparing root replacement; $C V G-B$, biological composite valve graft; $C V G-M$, mechanical composite valve graft.

study period, which are all specifically described in the manuscript. Distribution of each variable was summarized excluding missing values. For multivariable analyses, both propensity score and logistic regression for predicting outcomes, missing values were replaced with the mode in categorical variables and the median in continuous variables.

All continuous variables were tested for normality. Hypothesis of normality was rejected with all continuous variables but body mass index. When not otherwise specified, median with interquartile range, not mean with standard deviation, was used to represent distribution of continuous variables. As a measure of imbalance between groups, before and after matching, standardized mean difference (SMD) was used. For comparing values between unmatched treatment groups, the Mann-Whitney-Wilcoxon test was used for continuous variables and Pearson $\chi^{2}$ test for categorical variables. Between matched groups, a paired $t$ test was used for continuous variables and the McNemar test for categorical variables. Independent variables for logistic regression were selected based on existing literature and clinical experiences. Calculation of propensity scores and matching of cases based on propensity scores were conducted using $\mathrm{R}$ version 3.5.1 (R Foundation for Statistical Computing, Vienna, Austria) and MatchIt package version 3.0.2 (Daniel Ho and colleagues). All other statistical analyses were performed with JMP Pro Version 14.2.0 (SAS Institute Inc, Cary, NC). $P<.05$ was taken to indicate statistical significance.

\section{RESULTS}

\section{Preoperative Patient Characteristics}

Before matching, patients in the VSRR group were significantly younger (55 years) and had fewer cardiovascular risk factors than patients in the CVG group (65 years) (Table 1). In addition, more patients in the VSRR group suffered from MFS (47.1\%) than in the CVG group $(17.8 \%)$ (Table 1). In contrast, more patients in the CVG group suffered from AR grade III $(38.8 \%)$ or grade IV $(44.8 \%)$ and New York Heart Association class III or IV $(7.1 \%)$ than in the VSRR group $(29.7 \%, 36.2 \%$, and $2.7 \%$, respectively).
After matching, there were still similar but weaker differences in preoperative patient characteristics between the 2 groups (Table 1). Patients in the VSRR group were still younger with more MFS than those in the CVG group. A SMD plot indicating the bias reduction after matching is shown in Figure E2.

\section{Operative Variables}

Before matching, operation time, cardiopulmonary bypass time, and aortic crossclamping time were significantly longer in the VSRR group (411, 245, and $194 \mathrm{mi}-$ nutes, respectively) than in the CVG group $(399,223$, and 172 minutes, respectively, all $P<.01$ ). Aortic arch replacement, coronary artery bypass grafting $(\mathrm{CABG})$, and mitral valve surgery were performed significantly more frequently in the CVG group $(29.4 \%, 13.6 \%$, and $10.0 \%)$ than the VSRR group $(19.5 \%, 7.9 \%$, and $8.0 \%, P<.01, P<.01$, and $P<.05$, respectively). Homologous blood transfusion was performed more frequently in the CVG group (79.7\% vs $66.1 \%$, respectively, $P<.01$ ), whereas autologous blood transfusion was performed more frequently in the VSRR group $(22.0 \%$ vs $35.1 \%$, respectively, $P<.01)$ (Table 2).

After matching, cardiopulmonary bypass time and aortic crossclamping time were still significantly longer in the VSRR group (245 minutes and 193 minutes, respectively) than in the CVG group (223 minutes and 172 minutes, respectively, both $P<.01)$. Aortic arch replacement and CABG were still performed significantly more frequently in the CVG group $(29.5 \%$ and $13.4 \%)$ than the VSRR group $(19.6 \%$ and $8.1 \%$, both $P<.01$, respectively). The rates of homologous blood transfusion were still 
TABLE 1. Patients' clinical characteristics, per study group

\begin{tabular}{|c|c|c|c|c|c|c|c|}
\hline $\begin{array}{c}\text { Variable } \\
\mathbf{N}\end{array}$ & $\begin{array}{l}\text { Total } \\
3841\end{array}$ & $\begin{array}{c}\text { VSRR } \\
1345\end{array}$ & $\begin{array}{c}\text { CVG } \\
2496\end{array}$ & SMD & $\begin{array}{c}\begin{array}{c}\text { VSRR } \\
\text { (matched) }\end{array} \\
1155\end{array}$ & $\begin{array}{c}\begin{array}{c}\text { CVG } \\
\text { (matched) }\end{array} \\
1155\end{array}$ & $\begin{array}{c}\text { SMD } \\
\text { (matched) }\end{array}$ \\
\hline \multicolumn{8}{|l|}{ Year of operation } \\
\hline 2008-2009 & $434(11.3 \%)$ & $121(9.0 \%)$ & $313(12.5 \%)$ & -0.115 & $103(8.9 \%)$ & $117(10.1 \%)$ & -0.041 \\
\hline 2010-2011 & $562(14.6 \%)$ & $153(11.4 \%)$ & $409(16.4 \%)$ & -0.145 & $144(12.5 \%)$ & $135(11.7 \%)$ & 0.024 \\
\hline $2012-2013$ & $807(21.0 \%)$ & $269(20.0 \%)$ & $538(21.6 \%)$ & -0.038 & $241(20.9 \%)$ & $238(20.6 \%)$ & 0.006 \\
\hline 2014-2015 & $891(23.2 \%)$ & $324(24.1 \%)$ & $567(22.7 \%)$ & 0.032 & $266(23.0 \%)$ & $268(23.2 \%)$ & -0.004 \\
\hline 2016-2017 & $1147(29.9 \%)$ & $478(35.5 \%)$ & $669(26.8 \%)$ & 0.189 & $401(34.7 \%)$ & $397(34.4 \%)$ & 0.007 \\
\hline Age, (y) & $63(49-70)$ & $55(41-66)$ & $65(54-73)$ & -0.629 & $58(44-67)$ & $60(47-68)$ & -0.154 \\
\hline Sex (male) & $3036(79.0 \%)$ & $1049(78.0 \%)$ & $1987(79.6 \%)$ & -0.040 & $912(79.0 \%)$ & $930(80.5 \%)$ & -0.039 \\
\hline $\mathrm{BSA}, \mathrm{m}^{2}$ & $1.71(1.58-1.84)$ & $1.75(1.62-1.88)$ & $1.70(1.56-1.82)$ & 0.307 & $1.74(1.61-1.86)$ & $1.74(1.61-1.86)$ & 0.019 \\
\hline BMI & $22.6(20.2-25.0)$ & $22.6(20.0-25.1)$ & $22.6(20.3-25.0)$ & -0.035 & $22.8(20.2-25.3)$ & $22.9(20.5-25.3)$ & -0.088 \\
\hline Current smoker* & $435(11.3 \%)$ & $118(8.8 \%)$ & $317(12.7 \%)$ & -0.127 & $113(9.8 \%)$ & $125(10.8 \%)$ & -0.034 \\
\hline $\begin{array}{l}\text { Diabetes mellitus with } \\
\text { medication }\end{array}$ & $195(5.1 \%)$ & $67(5.0 \%)$ & $128(5.1 \%)$ & -0.007 & $65(5.6 \%)$ & $62(5.4 \%)$ & 0.011 \\
\hline Hypertension & $2538(66.1 \%)$ & $798(59.3 \%)$ & $1740(69.7 \%)$ & -0.219 & $720(62.3 \%)$ & $741(64.2 \%)$ & -0.039 \\
\hline Hyperlipidemia & $1028(26.8 \%)$ & $334(24.8 \%)$ & $694(27.8 \%)$ & -0.068 & $308(26.7 \%)$ & $318(27.6 \%)$ & -0.020 \\
\hline $\mathrm{CKD}^{\dagger}$ & $419(10.9 \%)$ & $119(8.8 \%)$ & $300(12.0 \%)$ & -0.104 & $114(9.9 \%)$ & $126(10.9 \%)$ & -0.034 \\
\hline Hemodialysis & $51(1.3 \%)$ & $17(1.3 \%)$ & $34(1.4 \%)$ & -0.009 & $16(1.4 \%)$ & $22(1.9 \%)$ & -0.041 \\
\hline COPD & $604(15.7 \%)$ & $207(15.4 \%)$ & $397(15.9 \%)$ & -0.014 & $175(15.2 \%)$ & $192(16.6 \%)$ & -0.041 \\
\hline Atrial fibrillation & $357(9.3 \%)$ & $86(6.4 \%)$ & $271(10.9 \%)$ & -0.160 & $82(7.1 \%)$ & $94(8.1 \%)$ & -0.039 \\
\hline Triple-vessel coronary disease & $52(2.1 \%)$ & $11(1.5 \%)$ & $41(2.4 \%)$ & -0.070 & $11(1.6 \%)$ & $14(1.9 \%)$ & -0.025 \\
\hline Angina pectoris $\mathrm{CCS} \geq 2$ & $74(1.9 \%)$ & $15(1.1 \%)$ & $59(2.4 \%)$ & -0.096 & $15(1.3 \%)$ & $17(1.5 \%)$ & -0.015 \\
\hline History of PCI & $108(2.8 \%)$ & $33(2.5 \%)$ & $75(3.0 \%)$ & -0.034 & $31(2.7 \%)$ & $30(2.6 \%)$ & 0.005 \\
\hline $\begin{array}{l}\text { Non-acute type A aortic } \\
\text { dissection }\end{array}$ & $291(7.6 \%)$ & $71(5.3 \%)$ & $220(8.8 \%)$ & -0.138 & $66(5.7 \%)$ & $66(5.7 \%)$ & 0.000 \\
\hline History of CVA & $215(5.6 \%)$ & $61(4.5 \%)$ & $154(6.2 \%)$ & -0.073 & $55(4.8 \%)$ & $61(5.3 \%)$ & -0.024 \\
\hline Carotid artery disease & $49(1.3 \%)$ & $11(0.8 \%)$ & $38(1.5 \%)$ & -0.066 & $11(1.0 \%)$ & $12(1.0 \%)$ & -0.009 \\
\hline PAD & $252(6.6 \%)$ & $72(5.4 \%)$ & $180(7.2 \%)$ & -0.077 & $65(5.6 \%)$ & $74(6.4 \%)$ & -0.033 \\
\hline Marfan syndrome & $500(27.7 \%)$ & $288(47.1 \%)$ & $212(17.8 \%)$ & 0.661 & $179(36.2 \%)$ & $152(30.4 \%)$ & 0.124 \\
\hline \multicolumn{8}{|l|}{ CHF NYHA class } \\
\hline Class III & $171(4.5 \%)$ & $28(2.1 \%)$ & $143(5.7 \%)$ & -0.189 & $28(2.4 \%)$ & $25(2.2 \%)$ & 0.017 \\
\hline Class IV & $43(1.1 \%)$ & $8(0.6 \%)$ & $35(1.4 \%)$ & -0.081 & $8(0.7 \%)$ & $5(0.4 \%)$ & 0.035 \\
\hline \multicolumn{8}{|l|}{ LVEF } \\
\hline$<60 \%$ & $1817(47.3 \%)$ & $526(39.1 \%)$ & $1291(51.7 \%)$ & -0.256 & $488(42.3 \%)$ & $496(43.0 \%)$ & -0.015 \\
\hline$<30 \%$ & $82(2.1 \%)$ & $22(1.6 \%)$ & $60(2.4 \%)$ & -0.055 & $21(1.8 \%)$ & $20(1.7 \%)$ & 0.006 \\
\hline \multicolumn{8}{|l|}{ Aortic regurgitation } \\
\hline Grade III & $1368(35.6 \%)$ & $400(29.7 \%)$ & $968(38.8 \%)$ & -0.192 & $382(33.1 \%)$ & $414(35.9 \%)$ & -0.060 \\
\hline Grade IV & $1605(41.8 \%)$ & $487(36.2 \%)$ & $1118(44.8 \%)$ & -0.176 & $454(39.3 \%)$ & $460(39.9 \%)$ & -0.012 \\
\hline Mitral regurgitation $\geq \mathrm{III}$ & $286(7.4 \%)$ & $75(5.6 \%)$ & $211(8.5 \%)$ & -0.113 & $67(5.8 \%)$ & $58(5.0 \%)$ & 0.034 \\
\hline Current $\beta$-blocker use & $983(26.2 \%)$ & $368(28.1 \%)$ & $615(25.2 \%)$ & 0.066 & $293(26.2 \%)$ & $290(25.7 \%)$ & 0.010 \\
\hline Use of anticoagulation & $38(1.0 \%)$ & $4(0.3 \%)$ & $34(1.4 \%)$ & -0.119 & $4(0.4 \%)$ & $4(0.4 \%)$ & 0.000 \\
\hline $\begin{array}{l}\text { Preoperative administration } \\
\text { of inotropic agents }\end{array}$ & $24(1.1 \%)$ & $1(0.1 \%)$ & $23(1.5 \%)$ & -0.155 & $1(0.1 \%)$ & $1(0.2 \%)$ & -0.001 \\
\hline
\end{tabular}


TABLE 1. Continued

\begin{tabular}{|c|c|c|c|c|c|c|c|}
\hline Variable & Total & VSRR & CVG & & $\begin{array}{c}\text { VSRR } \\
\text { (matched) }\end{array}$ & $\begin{array}{c}\text { CVG } \\
\text { (matched) }\end{array}$ & SMD \\
\hline $\mathbf{N}$ & 3841 & 1345 & 2496 & SMD & 1155 & 1155 & (matched) \\
\hline $\begin{array}{l}\mathrm{N} \text { cases VSRR and CVG replacement } \\
\geq 10 \text { per } \mathrm{y}\end{array}$ & $613(16.0 \%)$ & $323(24.0 \%)$ & $290(11.6 \%)$ & 0.328 & $236(20.4 \%)$ & $197(17.1 \%)$ & 0.087 \\
\hline
\end{tabular}

VSRR, Valve-sparing root replacement; $C V G$, composite valve graft; $S M D$, standardized mean difference; $B S A$, body surface area; $B M I$, body mass index; $C K D$, chronic kidney disease; $C O P D$, chronic obstructive pulmonary disease; $C C S$, Canadian Cardiovascular Society; $P C I$, percutaneous coronary intervention; $C V A$, cerebrovascular accident; $P A D$, peripheral arterial disease; $C H F$, congestive heart failure; $N Y H A$, New York Heart Association, $L V E F$, left ventricular ejection fraction. *Current smoker was defined as a patient with a history of smoking within 1 month before surgery. $\dagger$ Chronic kidney disease was defined as either detection of proteinuria or serum creatinine level $\geq 1.3$ mg/dL or estimate glomerular filtration rate $\leq 60 \mathrm{~mL} / \mathrm{min} / 1.73 \mathrm{~m}^{2}$ (Clinical Practice Guidebook for Diagnosis and Treatment of Chronic Kidney Disease 2009 published by the Japanese Society of Nephrology)

significantly greater in the CVG group $(73.6 \%)$ than the VSRR group $(68.7 \%, P<.01)$, whereas autologous blood transfusion was performed more frequently in the VSRR group $(23.8 \%$ vs $33.8 \%, P<.01)$ (Table 2 ).

\section{Postoperative Outcomes}

Before matching, the incidence of re-exploration for bleeding was similar between the groups (VSRR 3.6\%, CVG replacement $4.8 \%, P=.11$ ). The rate of postoperative stroke and hemodialysis was significantly greater in the CVG group $(2.7 \%, 2.3 \%)$ than the VSRR group $(1.0 \%$, $0.9 \%$, respectively, both $P<.01)$. The $\mathrm{CVG}$ group had a significantly longer mechanical ventilation time than the VSRR group (14 hours vs 11 hours, respectively, $P<.01$ ), although their distribution was highly skewed. Prolonged mechanical ventilation $>72$ hours was significantly more frequent in the CVG group than the VSRR group (7.4\% vs $4.3 \%$, respectively, $P<.01)$. Both intensive care unit and postoperative hospital stay were significantly longer in the CVG group ( 3 and 21 days) than the VSRR group ( 2 and 18 days, respectively, both $P<.01$ ). In-hospital mortality rates were significantly greater in the CVG group $(2.8 \%)$ than the VSRR group $(0.7 \%, P<.01)$ (Table 2$)$.

After matching, the incidence of re-exploration for bleeding was also similar between the groups (VSRR $3.5 \%$, CVG replacement $4.6 \%, P=.16$ ). Otherwise, there were no significant differences related to postoperative morbidity between the 2 groups. In-hospital mortality rates were still significantly greater in the CVG group $(1.8 \%)$ than the VSRR group $(0.8 \%)$ (Table 2$)$.

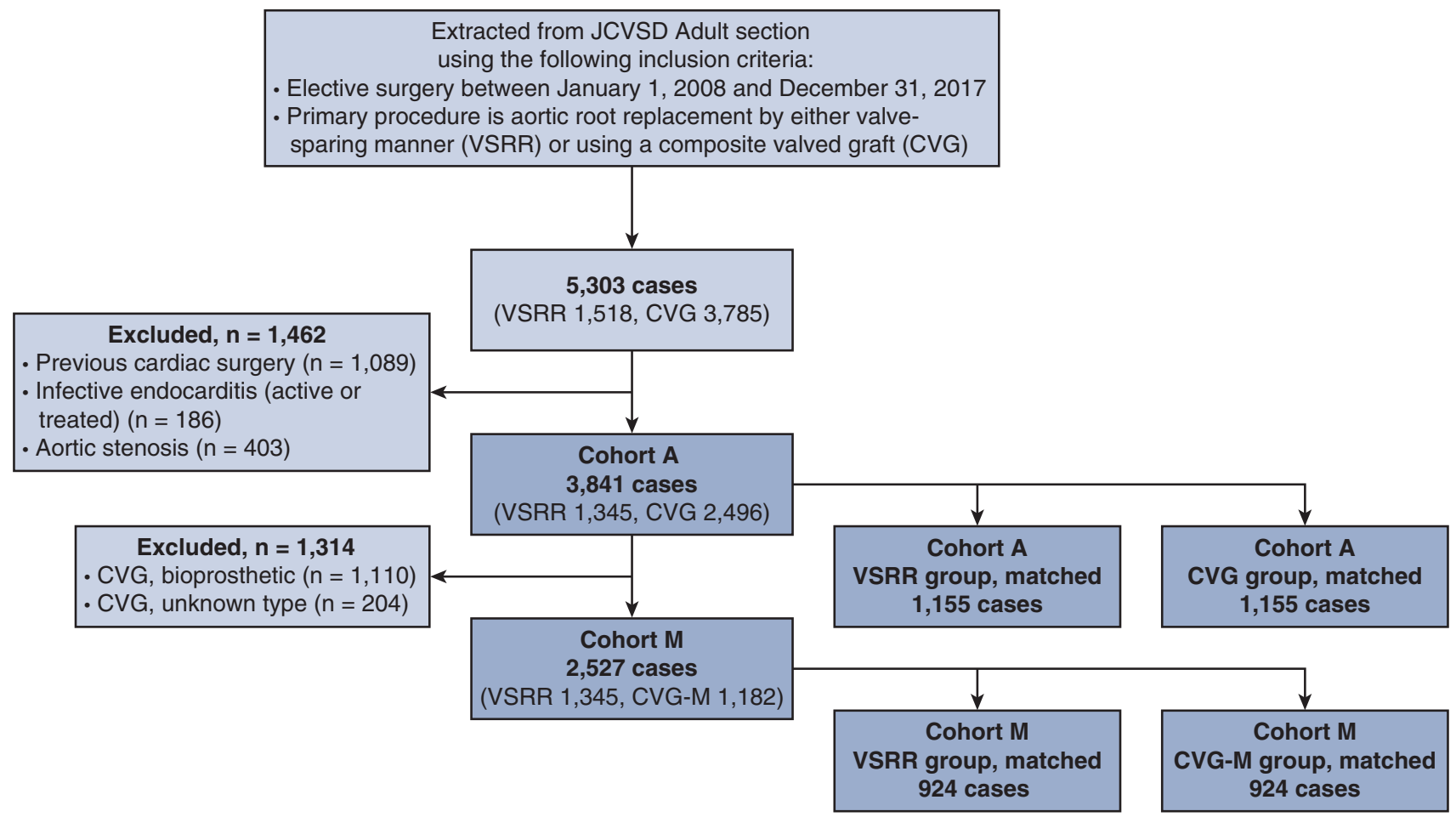

FIGURE 2. Consort type diagram of all patients undergoing either VSRR or CVG replacement. JCVSD, Japan Cardiovascular Surgery Database; VSSR, valve-sparing root replacement; $C V G$, composite valve graft; $C V G-M$, mechanical composite valve graft. 
TABLE 2. Intra- and postoperative variables, per study group

\begin{tabular}{|c|c|c|c|c|c|c|c|}
\hline$\frac{\text { Variable }}{\mathrm{N}}$ & $\begin{array}{l}\text { Total } \\
3841\end{array}$ & $\begin{array}{c}\text { VSRR } \\
1345\end{array}$ & $\begin{array}{l}\text { CVG } \\
2496\end{array}$ & $P$ value & $\begin{array}{c}\begin{array}{c}\text { VSRR } \\
\text { (matched) }\end{array} \\
1155\end{array}$ & $\begin{array}{c}\begin{array}{c}\text { CVG } \\
\text { (matched) }\end{array} \\
1155\end{array}$ & $\begin{array}{c}P \text { value } \\
\text { (matched) }\end{array}$ \\
\hline \multicolumn{8}{|l|}{ Intraoperative variables } \\
\hline Aortic arch replacement & $519(25.5 \%)$ & $156(19.5 \%)$ & $363(29.4 \%)$ & $<.01$ & $131(19.6 \%)$ & $196(29.5 \%)$ & $<.01$ \\
\hline Concomitant CABG & $445(11.6 \%)$ & $106(7.9 \%)$ & $339(13.6 \%)$ & $<.01$ & $93(8.1 \%)$ & $155(13.4 \%)$ & $<.01$ \\
\hline Concomitant mitral surgery & $356(9.3 \%)$ & $107(8.0 \%)$ & $249(10.0 \%)$ & .04 & $92(8.0 \%)$ & $87(7.5 \%)$ & .70 \\
\hline Concomitant mitral valve repair & $293(7.6 \%)$ & $105(7.8 \%)$ & $188(7.5 \%)$ & .76 & $90(7.8 \%)$ & $68(5.9 \%)$ & .07 \\
\hline Concomitant maze procedure & $69(1.8 \%)$ & $25(1.9 \%)$ & $44(1.8 \%)$ & .84 & $23(2.0 \%)$ & $15(1.3 \%)$ & .14 \\
\hline Homologous blood transfusion & $2879(75.0 \%)$ & $889(66.1 \%)$ & $1990(79.7 \%)$ & $<.01$ & $794(68.7 \%)$ & $850(73.6 \%)$ & $<.01$ \\
\hline Autologous blood transfusion & $1020(26.6 \%)$ & $472(35.1 \%)$ & $548(22.0 \%)$ & $<.01$ & $390(33.8 \%)$ & $275(23.8 \%)$ & $<.01$ \\
\hline Operation time, $\min$ & $403(330-500)$ & $411(345-496)$ & $399(320-501)$ & $<.01$ & $410(343-500)$ & $397(317-496)$ & .08 \\
\hline $\mathrm{CPB}$ time, $\min$ & $232(190-285)$ & $245(206-292)$ & $223(181-281)$ & $<.01$ & $245(205-292)$ & $223(180-275)$ & $<.01$ \\
\hline AXC time, $\min$ & $182(147-219)$ & $194(162-231)$ & $172(141-213)$ & $<.01$ & $193(160-231)$ & $172(148-220)$ & $<.01$ \\
\hline \multicolumn{8}{|l|}{ Postoperative variables } \\
\hline Postoperative stroke & $82(2.1 \%)$ & $14(1.0 \%)$ & $68(2.7 \%)$ & $<.01$ & $13(1.1 \%)$ & $21(1.8 \%)$ & .17 \\
\hline Re-exploration for bleeding & $168(4.4 \%)$ & $49(3.6 \%)$ & $119(4.8 \%)$ & .10 & $40(3.5 \%)$ & $53(4.6 \%)$ & .16 \\
\hline Postoperative hemodialysis & $70(1.8 \%)$ & $12(0.9 \%)$ & $58(2.3 \%)$ & $<.01$ & $12(1.0 \%)$ & $18(1.6 \%)$ & .26 \\
\hline Postoperative heart block & $60(1.6 \%)$ & $23(1.7 \%)$ & $37(1.5 \%)$ & .59 & $19(1.6 \%)$ & $18(1.6 \%)$ & .87 \\
\hline Postoperative atrial fibrillation & $997(26.0 \%)$ & $301(22.4 \%)$ & $696(27.9 \%)$ & $<.01$ & $283(24.5 \%)$ & $287(24.9 \%)$ & .85 \\
\hline Intubation time, $\mathrm{h}$ & $14(6-19)$ & $11(5-17)$ & $14(7-20)$ & $<.01$ & $11(5-17)$ & $14(6-20)$ & .17 \\
\hline Prolonged ventilation $>72 \mathrm{~h}$ & $242(6.3 \%)$ & $58(4.3 \%)$ & $184(7.4 \%)$ & $<.01$ & $55(4.8 \%)$ & $77(6.7 \%)$ & .05 \\
\hline ICU stay, d & $3(2-4)$ & $2(2-4)$ & $3(2-5)$ & $<.01$ & $2(2-4)$ & $3(2-5)$ & $<.01$ \\
\hline Postoperative hospital stay, $\mathrm{d}$ & $19(14-27)$ & $18(13-24)$ & $21(15-28)$ & $<.01$ & $18(13-24)$ & $20(15-27)$ & .21 \\
\hline In-hospital mortality & $80(2.1 \%)$ & $9(0.7 \%)$ & $71(2.8 \%)$ & $<.01$ & $9(0.8 \%)$ & $21(1.8 \%)$ & .02 \\
\hline
\end{tabular}

VSRR, Valve-sparing root replacement; $C V G$, composite valve graft; $C A B G$, coronary artery bypass grafting; $C P B$, cardiopulmonary bypass; $A X C$, aortic cross clamping, $I C U$, intensive care unit.

\section{Multivariable Logistic Regression Model to Predict In-Hospital Mortality}

A propensity score-adjusted multivariable logistic regression model was developed to identify independent predictors of in-hospital mortality (Table 3). CVG group, age equal to or older than 65 years, and chronic kidney disease (serum $\mathrm{Cr}>2.0 \mathrm{mg} / \mathrm{dL}$ ) were identified as independent predictors of in-hospital mortality.

\section{Subgroup Analysis}

Despite appropriate propensity score matching, there were still residual differences in preoperative patient characteristics between the 2 groups. This might be due to increased number of CVG-B replacement in the recent series, which tends to be performed on relatively elderly patients (Figure 1). Therefore, we performed subgroup analysis without patients who had CVG-B replacement.

TABLE 3. Odds ratios in a multivariable logistic regression model to predict in-hospital mortality

\begin{tabular}{|c|c|c|c|c|}
\hline & \multicolumn{4}{|c|}{ Odds ratios } \\
\hline & Estimate & 95\% CI lower limit & 95\% CI higher limit & $P$ value \\
\hline VSRR* & 0.06 & 0.01 & 0.31 & $<.01$ \\
\hline Propensity score & 0.08 & 0.00 & 4.54 & .22 \\
\hline Age $<50 \mathrm{y}^{\dagger}$ & 3.16 & 0.61 & 16.39 & .17 \\
\hline Age $\geq 65 \mathrm{y}^{\dagger}$ & 14.43 & 2.90 & 71.76 & $<.01$ \\
\hline Chronic kidney disease & 9.49 & 1.99 & 45.24 & $<.01$ \\
\hline CHF NYHA class $\geq$ III & 4.77 & 0.78 & 29.22 & .09 \\
\hline Aortic regurgitation $\geq$ III & 0.24 & 0.05 & 1.25 & .09 \\
\hline$N$ cases of VSRR and CVG replacement at hospital $\geq 10$ per $y$ & 0.04 & 0.00 & 2.24 & .12 \\
\hline
\end{tabular}

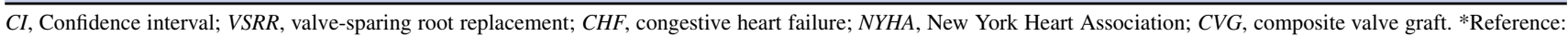
CVG. †Reference: $50 \mathrm{y} \leq$ age $<65 \mathrm{y}$. 
TABLE 4. Clinical characteristics of patients who underwent aortic root replacement except biological composite valve graft, per study group

\begin{tabular}{|c|c|c|c|c|c|c|c|}
\hline Variable & Total & VSRR & CVG-M & & $\begin{array}{c}\text { VSRR } \\
\text { (matched) }\end{array}$ & $\begin{array}{c}\text { CVG-M } \\
\text { (matched) }\end{array}$ & SMD \\
\hline $\mathbf{N}$ & 2527 & 1345 & 1182 & SMD & 924 & 924 & (matched) \\
\hline \multicolumn{8}{|l|}{ Year of operation } \\
\hline 2008-2009 & $303(12.0 \%)$ & $121(9.0 \%)$ & $182(15.4 \%)$ & -0.197 & $106(11.5 \%)$ & $122(13.2 \%)$ & -0.053 \\
\hline $2010-2011$ & $377(14.9 \%)$ & $153(11.4 \%)$ & $224(19.0 \%)$ & -0.212 & $125(13.5 \%)$ & $137(14.8 \%)$ & -0.037 \\
\hline 2012-2013 & $548(21.7 \%)$ & $269(20.0 \%)$ & $279(23.6 \%)$ & -0.087 & $194(21.0 \%)$ & $205(22.2 \%)$ & -0.029 \\
\hline 2014-2015 & $553(21.9 \%)$ & $324(24.1 \%)$ & $229(19.4 \%)$ & 0.115 & $208(22.5 \%)$ & $206(22.3 \%)$ & 0.005 \\
\hline 2016-2017 & $746(29.5 \%)$ & $478(35.5 \%)$ & $268(22.7 \%)$ & 0.286 & $291(31.5 \%)$ & $254(27.5 \%)$ & 0.088 \\
\hline Age, y & $56(43-65)$ & $55(41-66)$ & $56(45-64)$ & -0.089 & $56(43-65)$ & $56(45-64)$ & -0.091 \\
\hline Sex (male) & $2002(79.2 \%)$ & $1049(78.0 \%)$ & $953(80.6 \%)$ & -0.065 & $721(78.0 \%)$ & $733(79.3 \%)$ & -0.032 \\
\hline BSA, $\mathrm{m}^{2}$ & $1.75(1.62-1.88)$ & $1.75(1.62-1.88)$ & $1.75(1.61-1.88)$ & 0.038 & $1.75(1.62-1.88)$ & $1.75(1.61-1.88)$ & 0.017 \\
\hline BMI & $22.6(20.2-25.2)$ & $22.6(20.0-25.1)$ & $22.6(20.4-25.3)$ & -0.093 & $22.6(20.1-25.2)$ & $22.8(20.3-25.3)$ & -0.085 \\
\hline Current smoker* & $311(12.3 \%)$ & $118(8.8 \%)$ & $193(16.3 \%)$ & -0.230 & $101(10.9 \%)$ & $113(12.2 \%)$ & -0.041 \\
\hline $\begin{array}{l}\text { Diabetes mellitus with } \\
\text { medication }\end{array}$ & $122(4.8 \%)$ & $67(5.0 \%)$ & $55(4.7 \%)$ & 0.015 & $46(5.0 \%)$ & $44(4.8 \%)$ & 0.010 \\
\hline Hypertension & $1529(60.5 \%)$ & $798(59.3 \%)$ & $731(61.8 \%)$ & -0.051 & $548(59.3 \%)$ & $577(62.4 \%)$ & -0.064 \\
\hline Hyperlipidemia & $626(24.8 \%)$ & $334(24.8 \%)$ & $292(24.7 \%)$ & 0.003 & $232(25.1 \%)$ & $230(24.9 \%)$ & 0.005 \\
\hline $\mathrm{CKD}^{+}$ & $217(8.6 \%)$ & $119(8.8 \%)$ & $98(8.3 \%)$ & 0.020 & $79(8.5 \%)$ & $80(8.7 \%)$ & -0.004 \\
\hline Hemodialysis & $33(1.3 \%)$ & $17(1.3 \%)$ & $16(1.4 \%)$ & -0.008 & $13(1.4 \%)$ & $12(1.3 \%)$ & 0.009 \\
\hline COPD & $356(14.1 \%)$ & $207(15.4 \%)$ & $149(12.6 \%)$ & 0.080 & $124(13.4 \%)$ & $128(13.9 \%)$ & -0.013 \\
\hline Atrial fibrillation & $192(7.6 \%)$ & $86(6.4 \%)$ & $106(9.0 \%)$ & -0.097 & $67(7.3 \%)$ & $69(7.5 \%)$ & -0.008 \\
\hline Triple-vessel coronary disease & $28(1.9 \%)$ & $11(1.5 \%)$ & $17(2.3 \%)$ & -0.060 & $8(1.5 \%)$ & $10(1.7 \%)$ & -0.019 \\
\hline Angina pectoris $\mathrm{CCS} \geq 2$ & $47(1.9 \%)$ & $15(1.1 \%)$ & $32(2.7 \%)$ & -0.116 & $13(1.4 \%)$ & $12(1.3 \%)$ & 0.009 \\
\hline History of PCI & $56(2.2 \%)$ & $33(2.5 \%)$ & $23(1.9 \%)$ & 0.035 & $15(1.6 \%)$ & $18(1.9 \%)$ & -0.025 \\
\hline $\begin{array}{l}\text { Non-acute type A aortic } \\
\text { dissection }\end{array}$ & $198(7.8 \%)$ & $71(5.3 \%)$ & $127(10.7 \%)$ & -0.202 & $62(6.7 \%)$ & $73(7.9 \%)$ & -0.046 \\
\hline History of CVA & $115(4.6 \%)$ & $61(4.5 \%)$ & $54(4.6 \%)$ & -0.002 & $45(4.9 \%)$ & $42(4.5 \%)$ & 0.015 \\
\hline Carotid artery disease & $29(1.1 \%)$ & $11(0.8 \%)$ & $18(1.5 \%)$ & -0.066 & $9(1.0 \%)$ & $10(1.1 \%)$ & -0.011 \\
\hline PAD & $153(6.1 \%)$ & $72(5.4 \%)$ & $81(6.9 \%)$ & -0.063 & $53(5.7 \%)$ & $57(6.2 \%)$ & -0.018 \\
\hline Marfan syndrome & $455(35.1 \%)$ & $288(47.1 \%)$ & $167(24.3 \%)$ & 0.490 & $160(37.0 \%)$ & $137(28.8 \%)$ & 0.173 \\
\hline \multicolumn{8}{|l|}{ CHF NYHA class } \\
\hline Class III & $95(3.8 \%)$ & $28(2.1 \%)$ & $67(5.7 \%)$ & -0.187 & $28(3.0 \%)$ & $26(2.8 \%)$ & 0.013 \\
\hline Class IV & $25(1.0 \%)$ & $8(0.6 \%)$ & $17(1.4 \%)$ & -0.084 & $6(0.6 \%)$ & $9(1.0 \%)$ & -0.036 \\
\hline \multicolumn{8}{|l|}{ LVEF } \\
\hline$<60 \%$ & $1107(43.8 \%)$ & $526(39.1 \%)$ & $581(49.2 \%)$ & -0.203 & $398(43.1 \%)$ & $423(45.8 \%)$ & -0.054 \\
\hline$<30 \%$ & $52(2.1 \%)$ & $22(1.6 \%)$ & $30(2.5 \%)$ & -0.063 & $19(2.1 \%)$ & $15(1.6 \%)$ & 0.032 \\
\hline \multicolumn{8}{|l|}{ Aortic regurgitation } \\
\hline Grade III & $841(33.3 \%)$ & $400(29.7 \%)$ & $441(37.3 \%)$ & -0.161 & $319(34.5 \%)$ & $346(37.4 \%)$ & -0.061 \\
\hline Grade IV & $1027(40.6 \%)$ & $487(36.2 \%)$ & $540(45.7 \%)$ & -0.194 & $371(40.2 \%)$ & $388(42.0 \%)$ & -0.037 \\
\hline Mitral regurgitation $\geq$ III & $168(6.6 \%)$ & $75(5.6 \%)$ & $93(7.9 \%)$ & -0.092 & $57(6.2 \%)$ & $59(6.4 \%)$ & -0.009 \\
\hline Current $\beta$-blocker use & $664(27.0 \%)$ & $368(28.1 \%)$ & $296(25.6 \%)$ & 0.057 & $229(25.3 \%)$ & $243(27.0 \%)$ & -0.039 \\
\hline Use of anticoagulation & $17(0.7 \%)$ & $4(0.3 \%)$ & $13(1.1 \%)$ & -0.097 & $4(0.4 \%)$ & $5(0.6 \%)$ & -0.016 \\
\hline $\begin{array}{l}\text { Preoperative administration } \\
\text { of inotropic agents }\end{array}$ & $11(0.7 \%)$ & $1(0.1 \%)$ & $10(1.3 \%)$ & -0.136 & $1(0.2 \%)$ & $1(0.2 \%)$ & 0.001 \\
\hline
\end{tabular}


TABLE 4. Continued

\begin{tabular}{|c|c|c|c|c|c|c|c|}
\hline Variable & Total & VSRR & CVG-M & & $\begin{array}{c}\text { VSRR } \\
\text { (matched) }\end{array}$ & $\begin{array}{c}\text { CVG-M } \\
\text { (matched) }\end{array}$ & SMD \\
\hline $\mathbf{N}$ & 2527 & 1345 & 1182 & SMD & 924 & 924 & (matched) \\
\hline $\begin{array}{l}\mathrm{N} \text { cases VSRR and CVG replacement } \\
\geq 10 \text { per } \mathrm{y}\end{array}$ & $458(18.1 \%)$ & $323(24.0 \%)$ & $135(11.4 \%)$ & 0.334 & $154(16.7 \%)$ & $131(14.2 \%)$ & 0.069 \\
\hline
\end{tabular}

VSRR, Valve-sparing root replacement; $C V G-M$, mechanical composite valve graft; $S M D$, standardized mean difference; $B S A$, body surface area; $B M I$, body mass index; $C K D$, chronic kidney disease, COPD, chronic obstructive pulmonary disease; CCS, Canadian Cardiovascular Society; $P C I$, percutaneous coronary intervention; $C V A$, cerebrovascular accident; $P A D$, peripheral arterial disease; $C H F$, congestive heart failure; $N Y H A$, New York Heart Association; $L V E F$, left ventricular ejection fraction; $C V G$, composite valve graft. *Current smoker was defined as a patient with a history of smoking within 1 month before surgery. $\dagger$ Chronic kidney disease was defined as either detection of proteinuria or serum creatinine level $\geq 1.3 \mathrm{mg} / \mathrm{dL}$ or estimate glomerular filtration rate $\leq 60 \mathrm{~mL} / \mathrm{min} / 1.73 \mathrm{~m}^{2}$ (Clinical Practice Guidebook for Diagnosis and Treatment of Chronic Kidney Disease 2009 published by the Japanese Society of Nephrology).

We then matched patients who underwent VSRR with those who underwent CVG-M replacement in the same manner as Cohort A (a caliper width $=0.051425)($ Cohort $\mathrm{M}, \mathrm{n}=924$ each) (Table 4). Mirror histograms and a SMD plot are shown in Figures E3 and E4.

After matching, MFS was still more frequent in the VSRR group; however, most importantly, age was quite similar between the groups (both median 56 years old). Although aortic arch replacement was performed more frequently in the CVG-M group, other concomitant operations were performed with similar frequency. The same trends were observed with regard to transfusion and procedure time as Cohort A (Tables 4 and 5).

As results, the incidence of re-exploration for bleeding was also similar between groups (VSRR 3.9\%, CVG replacement $4.0 \%, P=.91)$. The incidence of postoperative stroke and prolonged mechanical ventilation was significantly greater and intensive care unit stay was significantly longer in the CVG-M group than the VSRR group. In-hospital mortality was still significantly greater in the CVG-M group $(2.8 \%)$ than the VSRR group $(0.8 \%, P<.01)$ (Table 5$)$.

\section{DISCUSSION}

The results of the present study indicated that many cardiovascular risk factors were more frequent in the CVG group, resulting in more frequent $\mathrm{CABG}$ performed in the CVG group. VSRR might be performed by more experienced surgeons and associated with a lower incidence of coronary anastomotic problems, which might be another reason for more frequent CABG performed in the CVG group. Furthermore, this might also be the reason why VSRR was associated with lower morbidity and mortality rates. Relatively longer duration of hospitalization in both groups, compared with many other countries, might be due to the Japanese insurance system.

After matching, procedure time was still significantly longer in the VSRR group than the CVG group, but postoperative morbidity rates were comparable between the 2 groups. In-hospital mortality rate was still significantly lower in the VSRR group.

In surgical intervention for aortic root aneurysm with or without AR, surgeons should select the most appropriate procedure according to the patient's background, with weighting for long-term (anticoagulation, durability) and early (bleeding, mortality) risks. VSRR seems an ideal solution to eliminate the need for anticoagulation treatment and to reduce future risks of reoperations compared with CVG-B replacement, although the follow-up periods of previous studies are still not sufficiently long and the mechanical Bentall procedure is still superior in terms of reoperation. ${ }^{9}$

Recent meta-analyses and large nationwide databases have revealed that the postoperative mortality rate after the Bentall procedure is around $6 \%$, which is greater than those after conventional cardiac operations. ${ }^{10-12}$ Common risk factors for early mortality were shown to be advanced age, urgent/emergent operation, concomitant mitral/ coronary disease, and longer procedure time. ${ }^{13-17}$ In contrast, bicuspid aortic valve and recent operation were identified as protective factors against early mortality. ${ }^{13}$ Therefore, when performed on an elective basis or for patients at low risk, the postoperative mortality rate decreased to $2.7 \%$ to $3.6 \%,{ }^{11,12,18}$ which was similar to the rate after conventional aortic valve replacement reported in the United States $(3.2 \%){ }^{19}$ When performed exclusively in young patients with MFS, postoperative mortality rate further decreased to $\leq 1 \% .^{20,21}$ In addition, operative mortality after aortic root replacement was reported to be better for greater-volume centers $(3.4 \%)$ than those with a lower volume $(5.8 \%))^{22}$

Operative mortality after VSRR was reported to be as low as $2 \%$ in a recent meta-analysis. ${ }^{23}$ There is no significant difference in early mortality between 2 major alternatives. ${ }^{24}$ Operative mortality also declines up to $1.4 \%$ to $1.88 \%$ on an elective basis or for patients at low risk in a database in the United States. ${ }^{7,18}$ When performed exclusively in patients with MFS in a high-volume center, VSRR could achieve a mortality rate of nearly $0 \%{ }^{25,26}$

Little evidence is available regarding comparisons of early outcomes of 2 homogeneous cohorts undergoing VSRR or CVG replacement. Table E1 summarizes reported series comparing early outcomes between the 2 approaches with sufficient numbers of patients ( $>50$ each). Although the results of the present study were derived from a 
TABLE 5. Intra- and postoperative variables of patients who underwent aortic root replacement except biological composite valve graft, per study group

\begin{tabular}{|c|c|c|c|c|c|c|c|}
\hline$\frac{\text { Variable }}{\mathrm{N}}$ & $\begin{array}{l}\text { Total } \\
2527\end{array}$ & $\begin{array}{c}\text { VSRR } \\
1345\end{array}$ & $\frac{\text { CVG-M }}{1182}$ & $P$ value & $\begin{array}{c}\begin{array}{c}\text { VSRR } \\
\text { (matched) }\end{array} \\
924\end{array}$ & $\begin{array}{c}\begin{array}{c}\text { CVG-M } \\
\text { (matched) }\end{array} \\
924\end{array}$ & $\begin{array}{c}P \text { value } \\
\text { (matched) }\end{array}$ \\
\hline \multicolumn{8}{|l|}{ Intraoperative variables } \\
\hline Aortic arch replacement & $311(23.9 \%)$ & $156(19.5 \%)$ & $155(31.2 \%)$ & $<.01$ & $89(17.8 \%)$ & $142(30.9 \%)$ & $<.01$ \\
\hline Concomitant CABG & $241(9.5 \%)$ & $106(7.9 \%)$ & $135(11.4 \%)$ & $<.01$ & $77(8.3 \%)$ & $97(10.5 \%)$ & .10 \\
\hline Concomitant mitral surgery & $211(8.3 \%)$ & $107(8.0 \%)$ & $104(8.8 \%)$ & .44 & $70(7.6 \%)$ & $70(7.6 \%)$ & 1 \\
\hline Concomitant mitral valve repair & $173(6.8 \%)$ & $105(7.8 \%)$ & $68(5.8 \%)$ & .04 & $68(7.4 \%)$ & $47(5.1 \%)$ & .05 \\
\hline Concomitant maze procedure & $43(1.7 \%)$ & $25(1.9 \%)$ & $18(1.5 \%)$ & .52 & $20(2.2 \%)$ & $15(1.6 \%)$ & .49 \\
\hline Homologous blood transfusion & $1761(69.7 \%)$ & $889(66.1 \%)$ & $872(73.8 \%)$ & $<.01$ & $621(67.2 \%)$ & $673(72.8 \%)$ & $<.01$ \\
\hline Autologous blood transfusion & $789(31.2 \%)$ & $472(35.1 \%)$ & $317(26.8 \%)$ & $<.01$ & $325(35.2 \%)$ & $252(27.3 \%)$ & $<.01$ \\
\hline Operation time, min & $407(334-500)$ & $411(345-496)$ & $401(321-503)$ & .02 & $411(345-505)$ & $400(319-496)$ & .26 \\
\hline $\mathrm{CPB}$ time, min & $238(196-290)$ & $245(206-292)$ & $227(181-287)$ & $<.01$ & $246(208-293)$ & $226(180-284)$ & $<.01$ \\
\hline AXC time, min & $189(153-229)$ & $194(162-231)$ & $181(144-221)$ & $<.01$ & $197(167-235)$ & $179(144-220)$ & $<.01$ \\
\hline \multicolumn{8}{|l|}{ Postoperative variables } \\
\hline Postoperative stroke & $44(1.7 \%)$ & $14(1.0 \%)$ & $30(2.5 \%)$ & $<.01$ & $12(1.3 \%)$ & $26(2.8 \%)$ & .02 \\
\hline Re-exploration for bleeding & $97(3.8 \%)$ & $49(3.6 \%)$ & $48(4.1 \%)$ & .59 & $36(3.9 \%)$ & $37(4.0 \%)$ & .91 \\
\hline Postoperative hemodialysis & $35(1.4 \%)$ & $12(0.9 \%)$ & $23(1.9 \%)$ & .02 & $9(1.0 \%)$ & $17(1.8 \%)$ & .10 \\
\hline Postoperative heart block & $43(1.7 \%)$ & $23(1.7 \%)$ & $20(1.7 \%)$ & .97 & $9(1.0 \%)$ & $14(1.5 \%)$ & .30 \\
\hline Postoperative atrial fibrillation & $572(22.6 \%)$ & $301(22.4 \%)$ & $271(22.9 \%)$ & .75 & $213(23.1 \%)$ & $203(22.0 \%)$ & .58 \\
\hline Intubation time, $\mathrm{h}$ & $12(6-18)$ & $11(5-17)$ & $14(7-20)$ & $<.01$ & $11(5-17)$ & $14(6-19)$ & .10 \\
\hline Prolonged ventilation $>72 \mathrm{~h}$ & $140(5.5 \%)$ & $58(4.3 \%)$ & $82(6.9 \%)$ & $<.01$ & $42(4.5 \%)$ & $65(7.0 \%)$ & .02 \\
\hline ICU stay, d & $3(2-4)$ & $2(2-4)$ & $3(2-5)$ & $<.01$ & $2(2-4)$ & $3(2-5)$ & $<.01$ \\
\hline Postoperative hospital stay, d & $19(14-26)$ & $18(13-24)$ & $20(15-28)$ & $<.01$ & $18(13-24)$ & $20(15-28)$ & .35 \\
\hline In-hospital mortality & $44(1.7 \%)$ & $9(0.7 \%)$ & $35(3.0 \%)$ & $<.01$ & $7(0.8 \%)$ & $26(2.8 \%)$ & $<.01$ \\
\hline
\end{tabular}

VSRR, Valve-sparing root replacement; $C V G-M$, mechanical composite valve graft; $C A B G$, coronary artery bypass grafting; $C P B$, cardiopulmonary bypass; $A X C$, aortic cross clamping; $I C U$, intensive care unit.

nationwide database accumulating data from more than 500 institutions over 10 years, in-hospital mortality $(<2 \%)$ rates of the 2 matched groups were excellent compared with experienced centers. Most importantly, VSRR was associated with lower in-hospital mortality compared with CVG replacement regardless of the prosthesis type. One of the reasons might be that VSRR had been performed favorably in the experienced, high-volume centers. Indeed, in lowvolume centers (caseload in the year $<10$ ), in-hospital mortality was significantly greater in the CVG group $(3.2 \%)$ than the VSRR group $(1.0 \%, P<.01)$, whereas the difference became less in high-volume centers (caseload in the year $\geq 10)($ CVG $0.9 \%$, VSRR $0 \%)$. It is unclear, however, in-hospital mortality became greater in selected patients undergoing CVG-M replacement alone, although the rate of cases performed in high-volume centers $(11.4 \%)$ was similar to that of Cohort A (11.6\%). CVG-M replacement for younger patients with low operative risk might favorably be performed by trainees; however, the experience of surgeon was not available in this database. Further study will be mandatory to clarify the impact of caseload.

Postoperative bleeding is another important issue. With the exception of reports including low-risk patients alone, some reports indicated a greater incidence of re-exploration for bleeding after CVG replacement
(3.1\%-8.0\%) than VSRR (3.1\%-5.9\%), ${ }^{8,18,27-29}$ whereas another multi-institutional cohort study yielded contradictory findings (CVG replacement: $5.3 \%$, VSRR: $6.9 \%){ }^{30}$ In the Society of Thoracic Surgeons database eliminating acute aortic dissection and infective endocarditis, the incidence of re-exploration for bleeding was quite similar for both methods (CVG replacement: 6.2\%-6.5\%, VSRR: $6 \%),{ }^{7}$ as well as in experienced high-volume centers in an elective/urgent setting alone (both 4\%). ${ }^{31}$ In the present study, both CVG and VSRR groups had lower incidences of postoperative bleeding $(4.1 \%$ and $3.6 \%$, respectively) compared with reported series, although this is a nationwide database study (Table 2 and Table E1). VSRR was not associated with a greater incidence of re-exploration for bleeding, but the incidence of homologous blood transfusion was lower than the other. This might have been due to the more frequent use of autologous blood in the VSRR group, which is believed to have more coagulability than packed red blood cell. In VSRR, most surgeons speculate that the risk of postoperative bleeding would be greater in remodeling than reimplantation because only one suture line is necessary for the former. However, in experienced institutions with caseloads $>100$, the incidence of re-exploration for bleeding was less frequent after remodeling $(2 \%)$ than after reimplantation $(3 \%-9 \%){ }^{32}$ It 
was unclear in our study which VSRR procedure was more likely to be responsible for postoperative bleeding. However, exclusively in 2017 , reimplantation was associated with lower incidence of re-exploration for bleeding $(3.6 \%)$ than remodeling $(6.4 \%)$, although difference was not significant $(P=.38)$.

Prolonged procedure time is one of the disadvantages of VSRR, although that of CVG replacement in this study was considerably longer than in reported series. In general, reimplantation requires a longer myocardial ischemia time than remodeling. ${ }^{32}$ According to our original nationwide survey, reimplantation was selected in two thirds of VSRR cases performed in Japan in $2014,{ }^{33}$ as well in the current study during 2017, which may explain the longer procedure time of VSRR in the present study. Indeed, cases of elective, isolated, and first-time remodeling with external suture annuloplasty require only $111 \pm 15$ minutes aortic crossclamping time $(n=15)$ in the first author's institution (data presented at the 70th Annual Scientific Meeting of the Japanese Association for Thoracic Surgery; September 27, 2017; Sapporo, Japan) (Video 1).

\section{Limitations}

The major limitation of this study was the lack of longterm follow-up data due to the inherent nature of this database. In general, CVG replacement is associated with lower risk of proximal reoperation but greater risk of other valverelated complications compared with VSRR. ${ }^{9}$ However, long-term valve durability after VSRR has been improving due to technical refinements over time. ${ }^{34,35}$ When long-term freedom from reoperation and survival become comparable between the groups, VSRR will be selected more often because early outcomes are better.

The second limitation was that only limited information in terms of operative procedure was available to us-details regarding the VSRR procedure, use of annuloplasty, use of cusp repair, and type of coronary re-attachment, etc, were unclear. This kind of information is essential to estimate the actual benefits of one approach over the other.

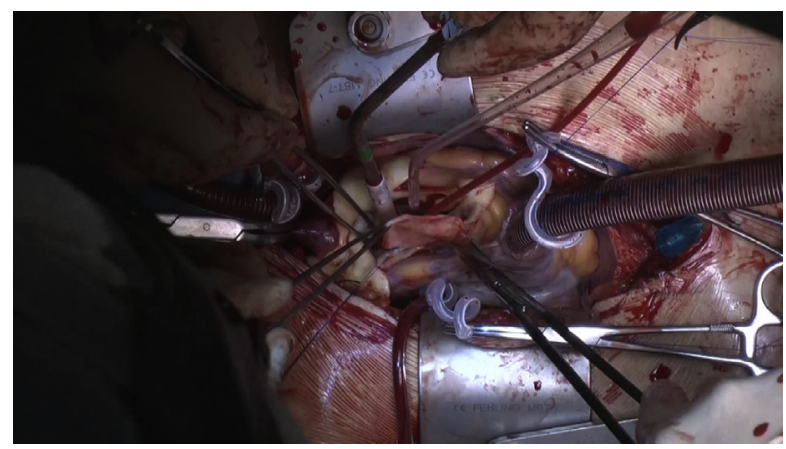

VIDEO 1. Aortic root remodeling procedure with external suture annuloplasty. Video available at: https://www.jtcvs.org/article/S00225223(19)30357-5/fulltext.
In addition, some of patient-specific echocardiographic and radiologic findings were either not collected or available only in recent years. Therefore, we were unable to perform subanalyses with regard to valve etiology, left ventricular dilatation, and proximal aortic dimension, etc. Some of these data are currently available in the latest version, so that further study must be made to clarify the impact of these factors.

The last limitation was residual small inhomogeneity between the groups even after subgroup analysis. We performed matching using baseline characteristics alone; therefore, there were still significant differences in concomitant procedures, which might have impact on the outcomes. Another subgroup analysis with isolated aortic root replacement alone should be performed to clarify this concern.

Despite these limitations, this study in which we analyzed data from more than 1000 propensity-matched patients enrolled in a Japanese nationwide database is clinically informative and will stimulate further clinical research. We hope that a prospective randomized trial for selected candidates will be launched in the near future to confirm the findings of the present study.

\section{CONCLUSIONS}

In overall Japanese institutions, VSRR was performed at a greater rate in patients at low risk and was thus associated with better morbidity and mortality rates compared with CVG replacement. After matching, both VSRR and CVG replacement were associated with excellent early postoperative mortality/morbidity rates in current Japanese centers for selected candidates, although VSRR was associated with lower morbidity and mortality rates despite longer procedure time.

\section{Conflict of Interest Statement}

Authors have nothing to disclose with regard to commercial support.

\section{References}

1. Wheat MW Jr, Wilson JR, Bartley TD. Successful replacement of the entire ascending aorta and aortic valve. JAMA. 1964;188:717-9.

2. Bentall H, De Bono A. A technique for complete replacement of the ascending aorta. Thorax. 1968;23:338-9.

3. David TE, Feindel CM. An aortic-valve sparing operation for patients with aortic incompetence and aneurysm of the ascending aorta. J Thorac Cardiovasc Surg. 1992;103:617-22.

4. Sarsam MA, Yacoub M. Remodeling of the aortic anulus. J Thorac Cardiovasc Surg. 1993;105:435-8.

5. David TE, David CM, Feindel CM, Manlhiot C. Reimplantation of the aortic valve at 20 years. J Thorac Cardiovasc Surg. 2017;153:232-8.

6. Schäfers HJ, Raddatz A, Schmied W, Takahashi H, Miura Y, Kunihara T, et al. Reexamining remodeling. J Thorac Cardiovasc Surg. 2015;149:S30-6.

7. Stamou SC, Williams ML, Gunn TM, Hagberg RC, Lobdell KW, Kouchoukos NT. Aortic root surgery in the United States: a report from the Society of Thoracic Surgeons database. J Thorac Cardiovasc Surg. 2015;149: 116-22.e4.

8. Gaudino M, Lau C, Munjal M, Avgerinos D, Girardi LN. Contemporary outcomes of surgery for aortic root aneurysms: a propensity-matched comparison 
of valve-sparing and composite valve graft replacement. J Thorac Cardiovasc Surg. 2015;150:1120-9.e1.

9. Benedetto U, Melina G, Takkenberg JJ, Roscitano A, Angeloni E, Sinatra R. Surgical management of aortic root disease in Marfan syndrome: a systematic review and meta-analysis. Heart. 2011;97:955-8.

10. Mookhoek A, Korteland NM, Arabkhani B, Di Centa I, Lansac E, Bekkers JA, et al. Bentall procedure: a systematic review and meta-analysis. Ann Thorac Surg. 2016;101:1684-9.

11. Williams JB, Peterson ED, Zhao Y, O'Brien SM, Andersen ND, Miller DC, et al. Contemporary results for proximal aortic replacement in North America. J Am Coll Cardiol. 2012;60:1156-62.

12. Dhurandhar V, Parikh R, Saxena A, Vallely MP, Wilson MK, Black DA, et al. The aortic root replacement procedure: 12-year experience from the Australian and New Zealand Society of Cardiac and Thoracic Surgeons Database. Heart Lung Circ. 2016;25:1245-51.

13. Di Marco L, Pacini D, Pantaleo A, Leone A, Barberio G, Marinelli G, et al. Composite valve graft implantation for the treatment of aortic valve and root disease: results in 1045 patients. J Thorac Cardiovasc Surg. 2016;152:1041-8.e1.

14. Woldendorp K, Starra E, Seco M, Hendel PN, Jeremy RW, Wilson MK, et al. Replacement of the aortic root with a composite valve-graft conduit: risk factor analysis in 246 consecutive patients. Heart Lung Circ. 2014;23:1187-93.

15. Schachner T, Vertacnik K, Nagiller J, Laufer G, Bonatti J. Factors associated with mortality and long time survival in patients undergoing modified Bentall operations. J Cardiovasc Surg (Torino). 2005;46:449-55.

16. Pacini D, Ranocchi F, Angeli E, Settepani F, Pagliaro M, Martin-Suarez S, et al. Aortic root replacement with composite valve graft. Ann Thorac Surg. 2003;76: 90-8.

17. Franke UF, Isecke A, Nagib R, Breuer M, Wippermann J, Tigges-Limmer K, et al. Quality of life after aortic root surgery: reimplantation technique versus composite replacement. Ann Thorac Surg. 2010;90:1869-75.

18. Caceres M, Ma Y, Rankin JS, Saha-Chaudhuri P, Englum BR, Gammie JS, et al. Mortality characteristics of aortic root surgery in North America. Eur J Cardiothorac Surg. 2014;46:887-93.

19. O'Brien SM, Shahian DM, Filardo G, Ferraris VA, Haan CK, Rich JB, et al; Society of Thoracic Surgeons Quality Measurement Task Force. The Society of Thoracic Surgeons 2008 cardiac surgery risk models: part 2isolated valve surgery. Ann Thorac Surg. 2009;88:S23-42.

20. Coselli JS, Volguina IV, LeMaire SA, Sundt TM, Connolly HM, Stephens EH, et al; Aortic Valve Operative Outcomes in Marfan Patients Study Group. Early and 1-year outcomes of aortic root surgery in patients with Marfan syndrome: a prospective, multicenter, comparative study. J Thorac Cardiovasc Surg. 2014; 147:1758-66.

21. Cameron DE, Alejo DE, Patel ND, Nwakanma LU, Weiss ES, Vricella LA, et al. Aortic root replacement in 372 Marfan patients: evolution of operative repair over 30 years. Ann Thorac Surg. 2009;87:1344-9.

22. Hughes GC, Zhao Y, Rankin JS, Scarborough JE, O'Brien S, Bavaria JE, et al. Effects of institutional volumes on operative outcomes for aortic root replacement in North America. J Thorac Cardiovasc Surg. 2013;145:166-70.
23. Arabkhani B, Mookhoek A, Di Centa I, Lansac E, Bekkers JA, De Lind Van Wijngaarden R, et al. Reported outcome after valve-sparing aortic root replacement for aortic root aneurysm: a systematic review and meta-analysis. Ann Thorac Surg. 2015;100:1126-31.

24. Liu L, Wang W, Wang X, Tian C, Meng YH, Chang Q. Reimplantation versus remodeling: a meta-analysis. J Card Surg. 2011;26:82-7.

25. Price J, Magruder JT, Young A, Grimm JC, Patel ND, Alejo D, et al. Long-term outcomes of aortic root operations for Marfan syndrome: a comparison of Bentall versus aortic valve-sparing procedures. J Thorac Cardiovasc Surg. 2016;151: 330-6.

26. Burgstaller JM, Held U, Mosbahi S, Stak D, Steurer J, Eckstein F, et al. A systemic review and meta-analysis: long-term results of the Bentall versus the David procedure in patients with Marfan syndrome. Eur J Cardiothorac Surg. 2018;54: 411-9.

27. Kallenbach K, Kojic D, Oezsoez M, Bruckner T, Sandrio S, Arif R, et al. Treat ment of ascending aortic aneurysms using different surgical techniques: a singlecentre experience with 548 patients. Eur J Cardiothorac Surg. 2013;44:337-45.

28. Esaki J, Leshnower BG, Binongo JN, Lasanajak Y, McPherson L, Halkos ME et al. Clinical outcomes of the David V valve-sparing root replacement compared with bioprosthetic valve-conduits for aortic root aneurysms. Ann Thorac Surg. 2017; 103:1824-32

29. Lee H, Cho YH, Sung K, Kim WS, Park KH, Jeong DS, et al. Clinical outcomes of root reimplantation and Bentall procedure: propensity score matching analysis. Ann Thorac Surg. 2018;106:539-47.

30. Lansac E, Bouchot O, Arnaud Crozat E, Hacini R, Doguet F, Demaria R, et al Standardized approach to valve repair using an expansible aortic ring versus mechanical Bentall: early outcomes of the CAVIAAR multicentric prospective cohort study. J Thorac Cardiovasc Surg. 2015;149:S37-45.

31. Zehr KJ, Orszulak TA, Mullany CJ, Matloobi A, Daly RC, Dearani JA, et al. Surgery for aneurysms of the aortic root: a 30-year experience. Circulation. 2004; 110:1364-71.

32. Kunihara T. Valve-sparing aortic root surgery. CON: remodeling. Gen Thorac Cardiovasc Surg. 2019;67:82-92.

33. Arimura S, Seki M, Sasaki K, Takai H, Matsuhama M, Kunihara T, et al. A nationwide survey of aortic valve surgery in Japan: current status of valve preservation in cases with aortic regurgitation. Gen Thorac Cardiovasc Surg. 2017; 65:429-34.

34. Ouzounian M, Rao V, Manlhiot C, Abraham N, David C, Feindel CM, et al. Valve-sparing root replacement compared with composite valve graft procedures in patients with aortic root dilation. J Am Coll Cardiol. 2016;68:1838-47.

35. Toeg H, Chan V, Rao RV, Chan KL, Ruel M, Mesana T, et al. Contemporary midterm echocardiographic outcomes of Bentall procedure and aortic valve sparing root replacement. Ann Thorac Surg. 2014;98:590-6.

Key Words: valve-sparing root replacement, composite valve graft replacement, database survey, postoperative outcome, aortic regurgitation, in-hospital mortality 
APPENDIX E1. FACTORS ENTERED INTO THE PROPENSITY SCORE MATCHING ANALYSIS TO ASSIGN PATIENTS TO UNDERGO VALVESPARING ROOT REPLACEMENT OVER COMPOSITE VALVE GRAFT REPLACEMENT

Age

Year of operation performed

Sex (male)

Body surface area

Body mass index

Current smoker

Chronic obstructive pulmonary disease

Diabetes mellitus with medication

Hypertension

Hyperlipidemia

Chronic kidney disease and hemodialysis

History of cerebrovascular accident

Carotid artery disease

Peripheral arterial disease

Marfan syndrome

Atrial fibrillation

Triple vessel coronary arterial disease
Congestive heart failure New York Heart Association class 0-II, III, IV

Canadian Cardiovascular Society angina class $\geq$ II

Left ventricular ejection fraction

Current $\beta$-blocker use

Current use of an anticoagulant drug

Preoperative administration of inotropic agents

Aortic regurgitation grade 0 -II, III, IV

Mitral regurgitation $\geq$ III

Non-acute type A aortic dissection

$\mathrm{N}$ cases valve-sparing root replacement and composite valve graft replacement $\geq 10$ per year

History of percutaneous coronary intervention

An interaction term between Marfan syndrome and age

\section{E-References}

E1. Miyata H, Tomotaki A, Motomura N, Takamoto S. Operative mortality and complication risk model for all major cardiovascular operations in Japan. Ann Thorac Surg. 2015;99:130-9.

E2. Ueki C, Miyata H, Motomura N, Sakata R, Sakaguchi G, Akimoto T, et al. Offpump technique reduces surgical mortality after elective coronary artery bypass grafting in patients with preoperative renal failure. J Thorac Cardiovasc Surg. 2018;156:976-83 


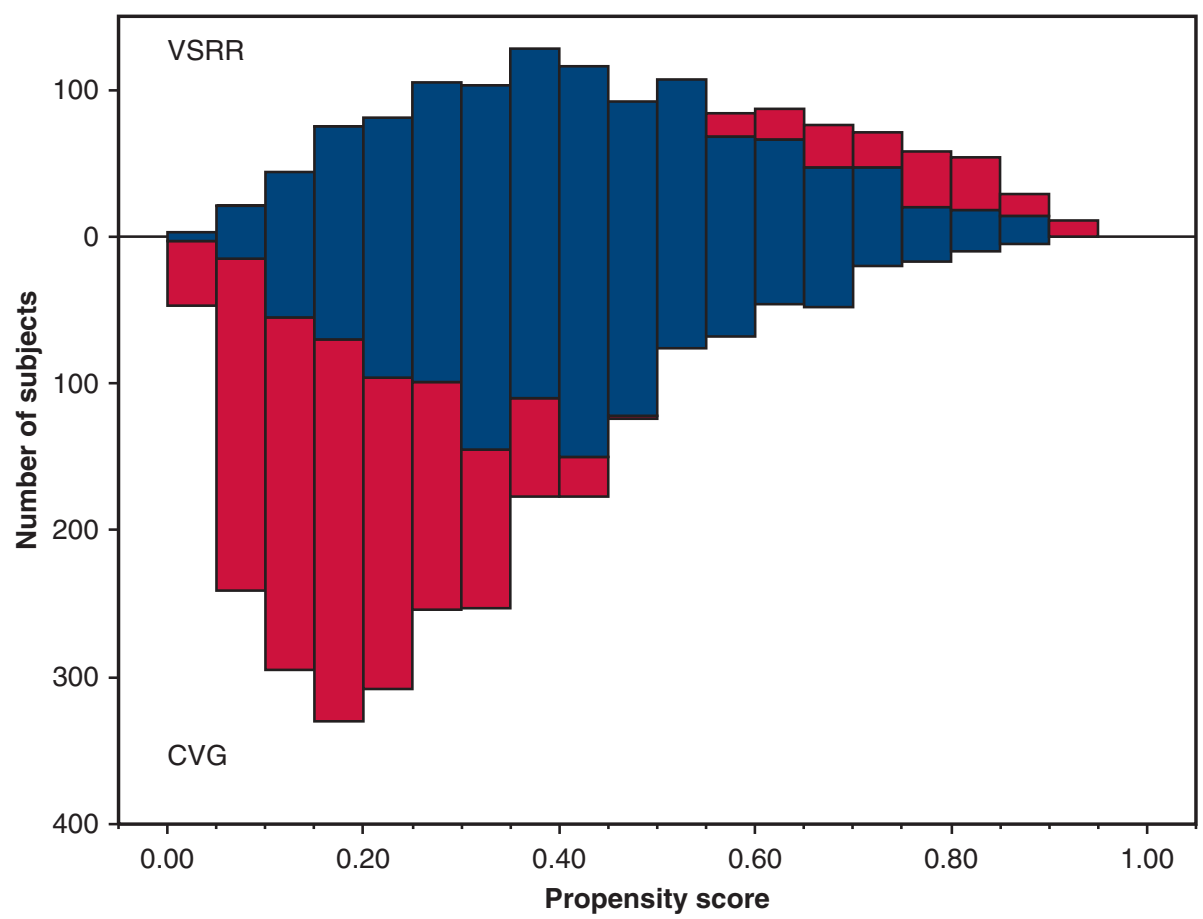

FIGURE E1. Mirror histogram showing the distribution of propensity scores for patients undergoing VSRR (bars above the zero line) or CVG replacement (bars below the zero line) before and after matching. Blue areas represent matched patient pairs, showing that they covered the complete spectrum of cases. $V S R R$, Valve-sparing root replacement; $C V G$, composite valve graft. 


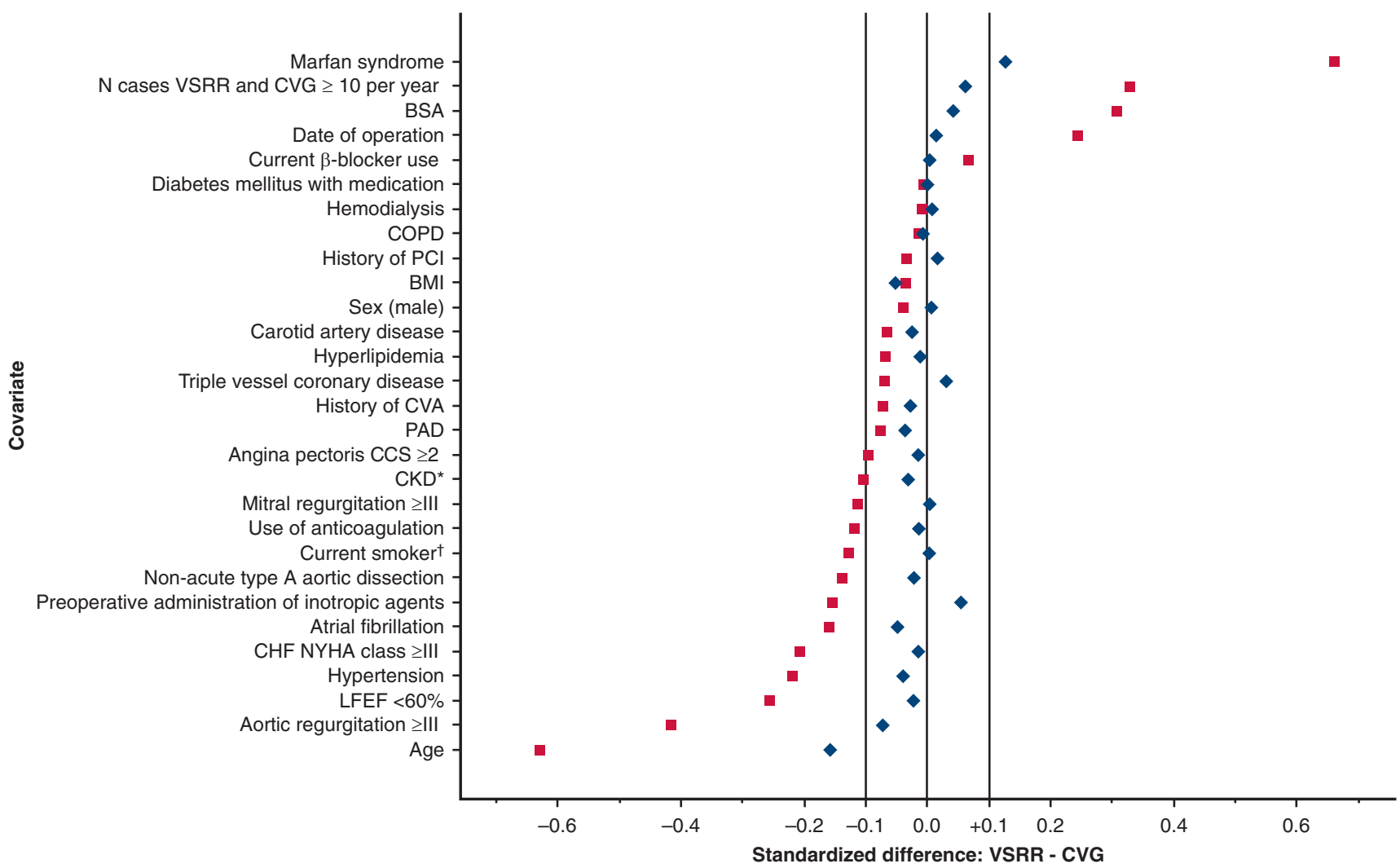

FIGURE E2. Covariate balance plot before (red squares) and after (blue diamonds) propensity score matching on selected covariates showing the bias reduction after matching. Symbols depict standardized differences for covariates between patients undergoing VSRR and CVG replacement. *CKD was defined as either detection of proteinuria or serum creatinine level $\geq 1.3 \mathrm{mg} / \mathrm{dL}$ or estimate glomerular filtration rate $\leq 60 \mathrm{~mL} / \mathrm{min} / 1.73 \mathrm{~m}{ }^{2}$ (Clinical Practice Guidebook for Diagnosis and Treatment of Chronic Kidney Disease 2009 published by the Japanese Society of Nephrology). †Current smoker was defined as a patient with a history of smoking within 1 month before surgery. VSRR, Valve-sparing root replacement; $C V G$, composite valve graft; BSA, body surface area; $C O P D$, chronic obstructive pulmonary disease; $P C I$, percutaneous coronary intervention; $B M I$, body mass index; $C V A$, cerebrovascular accident; $P A D$, peripheral arterial disease; $C C S$, Canadian Cardiovascular Society; $C K D$, chronic kidney disease; $C H F$, congestive heart failure; NYHA, New York Heart Association; $L V E F$, left ventricular ejection fraction. 


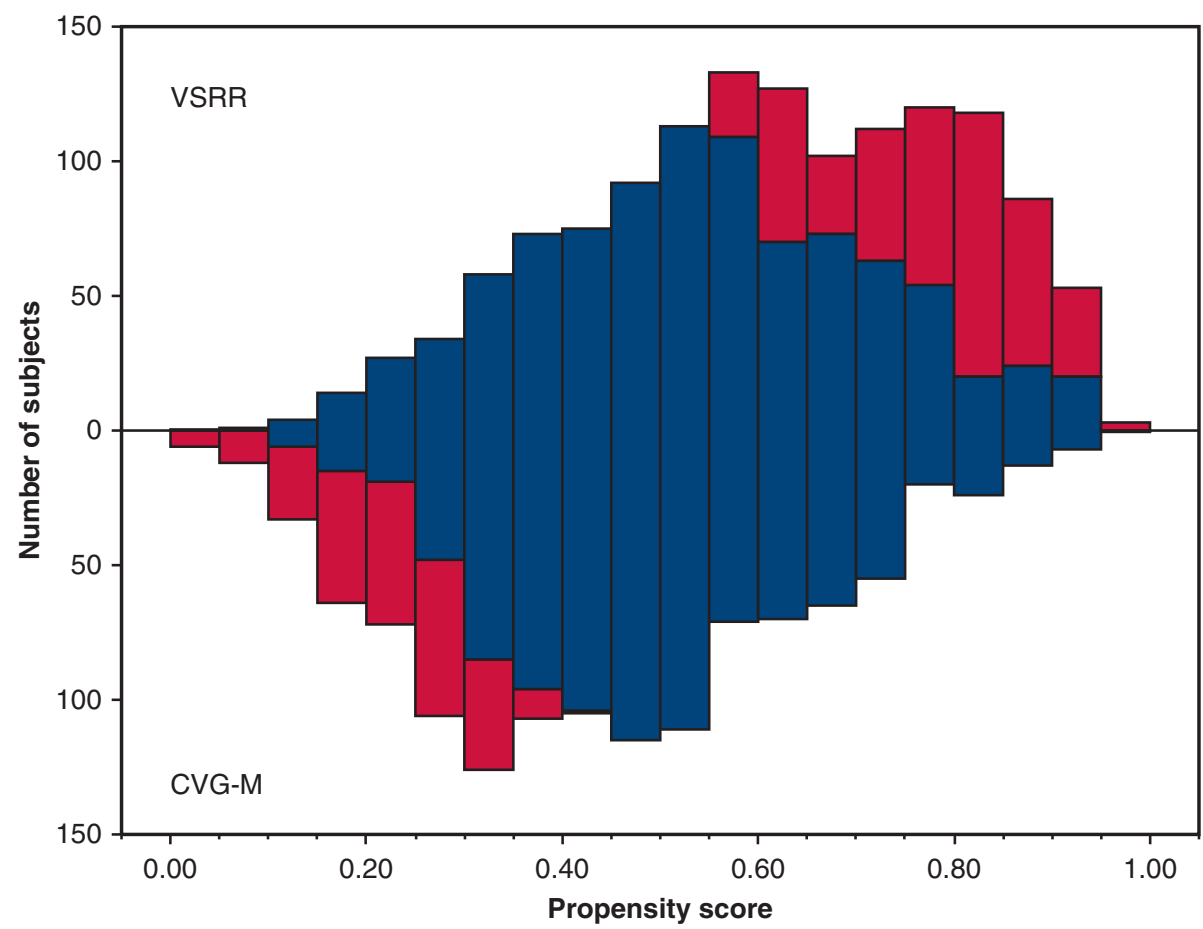

FIGURE E3. Mirror histogram showing the distribution of propensity scores for patients undergoing VSRR (bars above the zero line) or CVG-M replacement (bars below the zero line) before and after matching. Blue areas represent matched patient pairs, showing that they covered the complete spectrum of cases. VSRR, Valve-sparing root replacement; $C V G-M$, mechanical composite valve graft. 


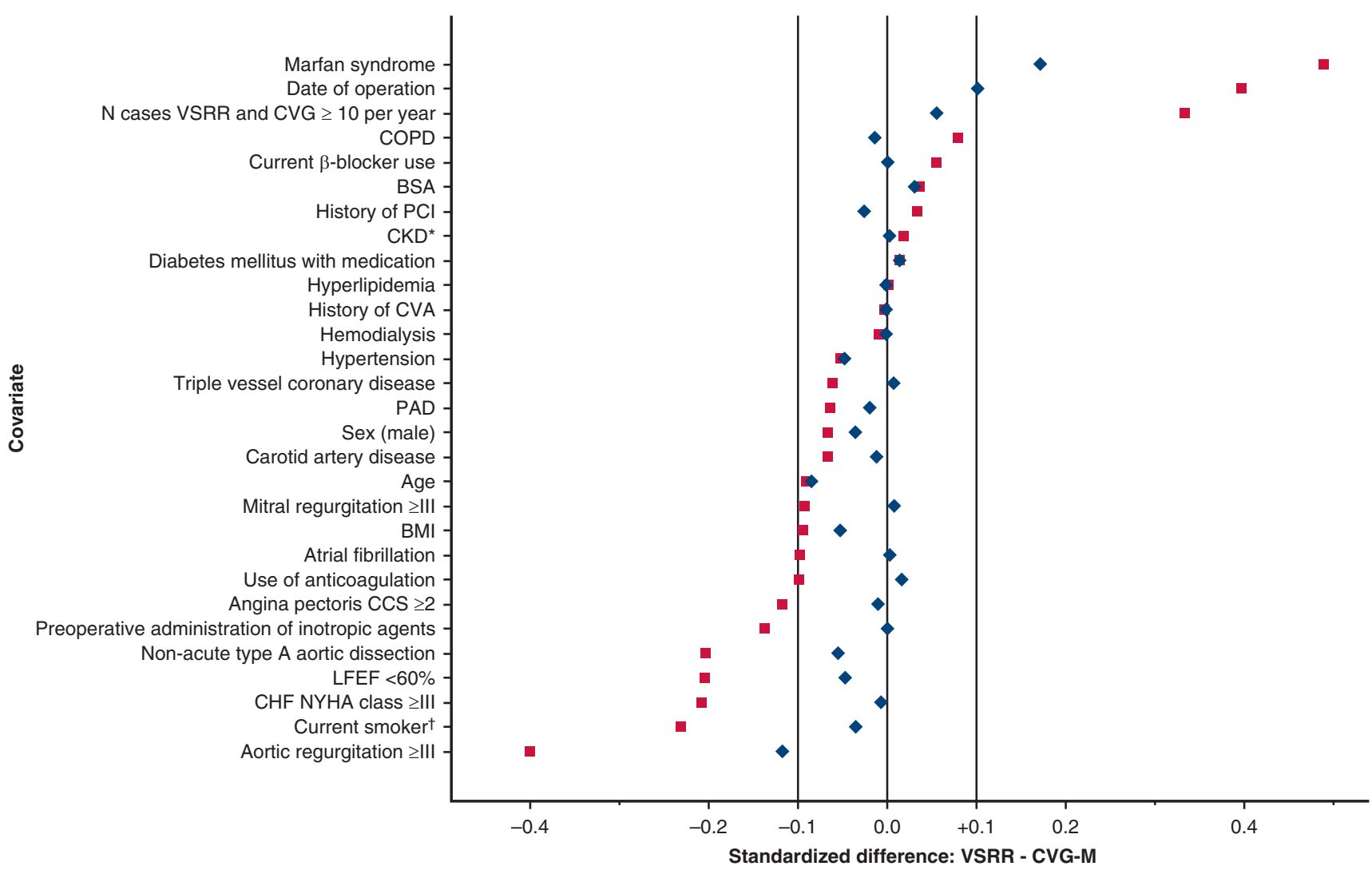

FIGURE E4. Covariate balance plot before (red squares) and after (blue diamonds) propensity score matching on selected covariates showing the bias reduction after matching. Symbols depict standardized differences for covariates between patients undergoing VSRR and CVG-M replacement. ${ }^{*} \mathrm{CKD}$ disease was defined as either detection of proteinuria or serum creatinine level $\geq 1.3 \mathrm{mg} / \mathrm{dL}$ or estimate glomerular filtration rate $\leq 60 \mathrm{~mL} / \mathrm{min} /$ $1.73 \mathrm{~m}^{2}$ (Clinical Practice Guidebook for Diagnosis and Treatment of Chronic Kidney Disease 2009 published by the Japanese Society of Nephrology). $\dagger$ Current smoker was defined as a patient with a history of smoking within 1 month before surgery. VSRR, Valve-sparing root replacement; $C V G$, composite valve graft; $C O P D$, chronic obstructive pulmonary disease; $B S A$, body surface area; $P C I$, percutaneous coronary intervention; $C K D$, chronic kidney disease; $C V A$, cerebrovascular accident; $P A D$, peripheral arterial disease; $B M I$, body mass index; $C C S$, Canadian Cardiovascular Society; $L V E F$, left ventricular ejection fraction; $C H F$, congestive heart failure; NYHA, New York Heart Association; $C V G-M$, mechanical composite valve graft. 
TABLE E1. Reported series comparing early outcomes between 2 approaches of aortic root replacement

\begin{tabular}{|c|c|c|c|c|c|c|c|c|c|c|c|}
\hline \multirow[b]{3}{*}{ Study } & \multicolumn{2}{|c|}{ Number of patients } & \multicolumn{4}{|c|}{ Mortality } & \multicolumn{2}{|c|}{$\begin{array}{c}\text { Re-exploration } \\
\text { for bleeding }\end{array}$} & \multirow{3}{*}{$\begin{array}{l}\text { Selection of } \\
\text { patients }\end{array}$} & \multirow[b]{3}{*}{ Source of patients } & \multirow{3}{*}{$\begin{array}{c}\text { Definition of } \\
\text { mortality }\end{array}$} \\
\hline & \multirow{2}{*}{$\begin{array}{c}\text { VSRR } \\
\text { (reimplantation) }\end{array}$} & \multirow{2}{*}{$\begin{array}{c}\text { CVG } \\
\text { (biological) }\end{array}$} & \multicolumn{2}{|c|}{$\begin{array}{c}\text { All } \\
\text { patients }\end{array}$} & \multicolumn{2}{|c|}{$\begin{array}{c}\text { Elective } \\
\text { cases alone }\end{array}$} & \multirow[b]{2}{*}{ VSRR } & \multirow[b]{2}{*}{ CVG } & & & \\
\hline & & & VSRR & CVG & $\overline{\text { VSRR }}$ & CVG & & & & & \\
\hline Zehr et al, $2004^{31}$ & $54(46)$ & $149(21)$ & & & $3.7 \%$ & $4.0 \%$ & $4.0 \%$ & $4.0 \%$ & $\begin{array}{l}\text { Elective/urgent cases } \\
\text { alone }\end{array}$ & Single institution & Operative \\
\hline Franke et al, $2010^{17}$ & $76(76)$ & 67 & $2.6 \%$ & $10.4 \%$ & & & & & & Three institutions & In-hospital \\
\hline Kallenbach et al, $2013^{27}$ & $96(83)$ & 298 & $1.0 \%$ & $4.8 \%$ & & & $3.1 \%$ & $6.0 \%$ & & Single institution & 30-day \\
\hline Caceres et al, $2014^{18}$ & 3585 & 28,162 & $4.5 \%$ & $8.9 \%$ & $1.4 \% *$ & $3.1 \% *$ & $5.2 \%$ & $7.6 \%$ & & STS database & Operative \\
\hline Coselli et al, $2014^{20}$ & $239(238)$ & 77 & $0.4 \%$ & $1.0 \%$ & & & $5.0 \%$ & $9.0 \%$ & $\begin{array}{l}\text { Marfan syndrome } \\
\text { international } \\
\text { registry }\end{array}$ & Single institution & 30-day \\
\hline Toeg et al, $2014^{35}$ & $68(68)$ & $96(34 \dagger)$ & & & $1.5 \%$ & $5.2 \%$ & & & & Single institution & In-hospital \\
\hline Stamou et al, $2015^{7}$ & 1918 & 4718 & & & $1.9 \%$ & $\begin{array}{c}3.8 \% \ddagger \\
\text { or } 5.7 \% \oint\end{array}$ & $6.0 \%$ & $\begin{array}{c}6.2 \% \ddagger \\
\text { or } 6.5 \% \oint\end{array}$ & $\begin{array}{l}\text { No acute aortic } \\
\text { dissection, no } \\
\text { infective } \\
\text { endocarditis }\end{array}$ & STS database & Operative \\
\hline Gaudino et al, $2015^{8}$ & $180(158)$ & $710(421)$ & $0.0 \%$ & $\begin{array}{c}0.3 \% \ddagger \\
\text { or } 0.2 \% \S\end{array}$ & & & $3.9 \%$ & $\begin{array}{c}3.1 \% \ddagger \\
\text { or } 3.6 \% \oint\end{array}$ & & Single institution & In-hospital \\
\hline Lansac et al, $2015^{30}$ & $130(0)$ & 131 & & & $3.8 \%$ & $3.8 \%$ & $6.9 \%$ & $5.3 \%$ & $\begin{array}{l}\text { CAVIAAR registry } \\
\text { (elective alone) }\end{array}$ & 20 institutions & 30-day \\
\hline Price et al, $2016^{25}$ & $98(69)$ & 67 & $0.0 \%$ & $0.0 \%$ & & & $2.0 \%$ & $1.5 \%$ & $\begin{array}{l}\text { Marfan syndrome } \\
\text { alone }\end{array}$ & 19 institutions & In-hospital \\
\hline Ouzounian et al, $2016^{34}$ & $253(200)$ & $363(180)$ & & & $0.4 \%$ & $\begin{array}{r}0.5 \% \ddagger \\
\text { or } 0 \% \S\end{array}$ & $5.9 \%$ & $\begin{array}{c}7.1 \% \ddagger \\
\text { or } 6.1 \% \S\end{array}$ & & Single institution & In-hospital \\
\hline Esaki et al, $2017^{28}$ & $282(282)$ & $425(425)$ & $2.8 \%$ & $9.6 \%$ & & & $5.7 \%$ & $8.0 \%$ & & Single institution & Operative \\
\hline Lee et al, $2018^{29}$ & $82(82)$ & 134 & $0.0 \%$ & $1.5 \%$ & & & $3.7 \%$ & $6.7 \%$ & & Single institution & Operative \\
\hline
\end{tabular}

